\title{
Review gPCE-Based Stochastic Inverse Methods: A Benchmark Study
from a Civil Engineer's Perspective
}

\author{
Filippo Landi ${ }^{1}\left(\mathbb{D}\right.$, Francesca Marsili $^{2}$, Noemi Friedman $^{3}$ and Pietro Croce ${ }^{1, *(\mathbb{D}}$ \\ 1 Department of Civil and Industrial Engineering, University of Pisa, Largo Lucio Lazzarino 1, 56122 Pisa, Italy; \\ filippo.landi@ing.unipi.it \\ 2 Federal Waterways Engineering and Research Institute, Kußmaulstraße 17, 76187 Karlsruhe, Germany; \\ francesca.marsili@baw.de \\ 3 Institute for Computer Science and Control, Kende u. 13, 1111 Budapest, Hungary; \\ friedman.noemi@sztaki.hu \\ * Correspondence: p.croce@ing.unipi.it; Tel.: +39-335-5345611
}

Citation: Landi, F.; Marsili, F.; Friedman, N.; Croce, P. gPCE-Based Stochastic Inverse Methods: A Benchmark Study from a Civil Engineer's Perspective. Infrastructures 2021, 6, 158. https://doi.org/ $10.3390 /$ infrastructures6110158

Academic Editors: Reza Haghani, Rami Hawileh and Paolo S. Valvo

Received: 17 September 2021

Accepted: 4 November 2021

Published: 5 November 2021

Publisher's Note: MDPI stays neutral with regard to jurisdictional claims in published maps and institutional affiliations.

Copyright: (c) 2021 by the authors. Licensee MDPI, Basel, Switzerland. This article is an open access article distributed under the terms and conditions of the Creative Commons Attribution (CC BY) license (https:/ / creativecommons.org/licenses/by/ $4.0 /)$.

\begin{abstract}
In civil and mechanical engineering, Bayesian inverse methods may serve to calibrate the uncertain input parameters of a structural model given the measurements of the outputs. Through such a Bayesian framework, a probabilistic description of parameters to be calibrated can be obtained; this approach is more informative than a deterministic local minimum point derived from a classical optimization problem. In addition, building a response surface surrogate model could allow one to overcome computational difficulties. Here, the general polynomial chaos expansion (GPCE) theory is adopted with this objective in mind. Owing to the fact that the ability of these methods to identify uncertain inputs depends on several factors linked to the model under investigation, as well as the experiment carried out, the understanding of results is not univocal, often leading to doubtful conclusions. In this paper, the performances and the limitations of three gPCE-based stochastic inverse methods are compared: the Markov Chain Monte Carlo (MCMC), the polynomial chaos expansion-based Kalman Filter (PCE-KF) and a method based on the minimum mean square error (MMSE). Each method is tested on a benchmark comprised of seven models: four analytical abstract models, a one-dimensional static model, a one-dimensional dynamic model and a finite element (FE) model. The benchmark allows the exploration of relevant aspects of problems usually encountered in civil, bridge and infrastructure engineering, highlighting how the degree of non-linearity of the model, the magnitude of the prior uncertainties, the number of random variables characterizing the model, the information content of measurements and the measurement error affect the performance of Bayesian updating. The intention of this paper is to highlight the capabilities and limitations of each method, as well as to promote their critical application to complex case studies in the wider field of smarter and more informed infrastructure systems.
\end{abstract}

Keywords: bayesian inversion; gPCE; surrogate model; uncertainty quantification; non-linear filter; parameter identification

\section{Introduction}

In recent decades, increasing effort has been devoted to the identification of constructed systems. Structural identification (St-Id) is actually a subfield of system identification, which originated in electrical engineering in relation to circuit and control theory; it has been defined as "the parametric correlation of structural response characteristics predicted by a mathematical model with analogous quantities derived from experimental measurements" [1].

Models that might be updated can be classified in two main classes: physics-based models, such as mathematical physics and discrete geometric models and non-physicsbased models, such as inter alia probabilistic, statistical and meta-models. 
Discrete geometric models include finite element (FE) models, commonly used in civil, bridge and structural engineering to analyze, e.g., the internal forces and displacements of structures in several limit states, or to predict the response of the system to dynamic actions such as earthquakes, wind and traffic. It must be emphasized, on the one hand, that the input parameters of the model (material properties, geometric properties, boundary conditions, load conditions, etc.) are affected by different sources of uncertainty; on the other, that simplifying modelling assumptions regarding the model structure are often required or implicitly made. All these issues may significantly decrease the quality and accuracy of the numerical model, which consequently needs suitable updating.

To improve the model, a possible technique relies on direct measurements of the parameter of interest; however, this approach is often hindered by several difficulties. For example, when assessing material parameters of existing structures, only a few nondestructive or semi-destructive in situ tests can be performed, due to the necessity to safeguard integrity and static performances of the construction [2-4]. Another frequently used approach consists of collecting static and dynamic information about the global and local behavior of the built structure of interest in its service state; to assess the structural response, particularly relevant parameters are, inter alia, strains, displacements, acceleration, time histories, frequency response functions, natural frequencies, mode shapes, etc. The latter approach is usually less intrusive, since experiments can often be executed in an operational state of the structure.

The uncertain input parameters of the model can be thus identified resorting to model updating techniques, also called model calibration or parameter estimation and identification.

Considering the updating procedure, a classical approach is to try to match the response of the updated model and the experimental data [5,6], minimizing the discrepancy between them. This discrepancy is evaluated by means of the so-called objective function. The values of the relevant parameters are indeed determined by minimizing the objective function with a suitable optimization algorithm. A problem that is frequently encountered with this approach is that the inversion problem-the task to compute the input parameters from the measured output-is not necessarily a well-posed one. There may be many sets of the parameter values that explain the observation in a "best" way and we restrict ourselves to finding some local minima of the problem. To obtain a unique solution, one may need to resort to a somewhat ad hoc regularization procedure [7].

The relevant input parameters might be considered as deterministic quantities, but, if previous information regarding their uncertainty is available, it is more suitable to describe these parameters as random variables, characterized by appropriate probability distributions that disclose uncertainties about the value of these input parameters. The calibration process of these parameters is tackled here in a probabilistic fashion, with the help of the Bayes theorem $[8,9]$ and the result of the identification is not a deterministic value of the parameters but an updated version of the a priori defined distributions. Furthermore, such a model can take into account both the confidence level of the input parameters and the confidence level of the measured responses.

Pioneering applications of the Bayesian inference methods for structural identification are in [10-13], while new examples can be found in [14-16]. Comprehensive reviews of this topic are given in [17-19]. The idea of probabilistic inversion is only now becoming more widespread in the civil engineering community. Nevertheless, some very promising applications for the calibration of FE model parameters are presented in [20], for a r.c. water tank; in [21], for a concrete gravity dam; in [22]; for a cable-stayed footbridge using dynamic measurements; and in [23], for a tall timber building. With such methods, not only parameters but also uncertain fields can be identified; for example, the compressibility field of soil below a gas platform is identified in [24] while a Bayesian hierarchical model is presented in [25] to characterize the spatial behavior of climate extremes. 
In recent years, research has widely focused on the computational aspects of the Bayesian inverse method [26]. The brute force approach is represented by the Markov Chain Monte Carlo (MCMC) method [27-30]. This approach has the advantage of being model-independent, but it requires a huge number of model simulations. The MCMC generates samples of the Bayesian posterior distribution of the parameters by a random walk, but, at every random step of the walk, the model response has to be evaluated at a new value of the parameters, which is computationally expensive. To speed up the process, it is possible to resort to a surrogate model of the system response, which can be obtained through a general polynomial chaos expansion (gPCE) of the model response [31-34]. Here, the idea is to replace the computationally expensive physics-based model-the FE modelwith a computationally inexpensive non-physics-based model—the surrogate modelcarrying out the updating of the first through the updating of the second. Applications of the gPCE-based stochastic inverse method for structural identification can be found in [20-24,35-44].

However, despite the implementation of the surrogate model, the MCMC is still affected by a low convergence rate; furthermore, it would be desirable to have a functional representation of the updated parameters instead of only obtaining samples of it. This can be especially important when the updating process has to be sequentially updated as new measurements become available. The Polynomial Chaos Expansion-based Kalman Filter (PCE-KF) $[45,46]$ is a linear Bayesian filter in which all random variables, the prior input parameters, the measurable outputs as well as the updated input parameters are functionally approximated, enabling a sampling-free updating. Nonetheless, the applicability of this method is mainly restricted to linear models. A recently proposed method based on the Minimum Mean Squared Estimator (MMSE) [47-49] overcomes this limitation by using a non-linear filter; this method is also explained and investigated in this paper. Evidently, the advantage of the used functional representation becomes more relevant as the complexity of the given construction, or of the given infrastructure, increases.

\section{Scope of the Overview}

As highlighted in the introduction, the application of Bayesian inverse methods has grown rapidly in recent years in many research fields, including structural identification. However, Bayesian inverse methods are often seen as "perfect" tools and applied without paying so much attention to the peculiarities of the investigated problem and the capabilities of the adopted updating procedure.

The aim of this work is to familiarize civil engineers with gPCE-based Bayesian inversion methods through "toy models" and simple civil engineering examples. In presenting and discussing the results obtained with different updating procedures for these simple examples, the capabilities of the different updating procedures are shown, focusing on the expected outcomes of the updating, as well as on the interpretation and skilled use of results. Keeping this in mind and without pretending to be exhaustive, we would like to draw attention to a few aspects of the St-Id problem, especially those that are more relevant from a civil engineering perspective [50]. In other words, we focus on the degree of non-linearity of the model, the engineering knowledge that is reflected by the adopted prior distribution model, the number of random variables involved in the problem, the information content of the available measurements and the magnitude of the measurement error. The aim is to promote a critical approach to the use of the mentioned gPCE-based updating procedures by first testing them on simplified models and then by moving to more complex and realistic case studies. There are seven relevant benchmark models considered in this paper: 
- Four analytical, abstract toy models;

- A one-dimensional static model (a clamped beam);

- A one-dimensional dynamic model (an inverse pendulum);

- An FE model of a real structure (a reinforced concrete water tank).

The updating procedures presented in the introduction, namely, the PCE-based MCMC, the PCE-KF and the method based on the MMSE, are tested on the benchmark; artificial measurements are generated, and the performance of each method is assessed. In this way, this study identifies the capabilities and limitations of each method, which is a useful approach for civil engineering. In fact, the correct use of updating procedures can provide evidence for informed decision making not only at the reliability assessment stage, but also during the design and maintenance planning of engineering structures and infrastructures [20].

In the following section, the Bayesian approach to the inverse problem is introduced and the numerical procedures are described in detail; then, the benchmark study is carried out and the partial outcome of each analysis is discussed, leading us to draw some promising conclusion.

\section{Methods}

\subsection{The Bayesian Approach to the Inverse Problem}

Let us consider a mechanical system whose behavior is modelled by a set of governing equations, i.e., partial differential equations. Let us suppose that the mechanical model is characterized by a vector $Q: \Omega \rightarrow R^{k}$ of input random parameters, with $\Omega$ the set of possible events. The epistemic uncertainty coming from our lack of knowledge is modelled by this randomness and we assign some arbitrary prior probability distribution $\pi_{Q}(\boldsymbol{q})$ of the $Q$ random vector, based on engineering judgement and professional expertise. We note one realization of $Q$ by $\boldsymbol{q} \in R^{k}$. Further in the paper, capital letters are used for random variables and small letters for their realizations. For mathematical convenience, we also introduce a map $F: R^{k} \rightarrow R^{n}$, which maps this set of random variables into a set of mutually independent random variables $Z: \Omega \rightarrow R^{n}$, characterized by elements $Z_{i}$, each one characterized by a standard normal distribution $\pi_{i}\left(z_{i}\right)$. We suppose that the $F$ map is a bijective one. Due to the independence, the joint prior density function of $\boldsymbol{Z}$ reads

$$
\pi_{\mathbf{Z}}(\boldsymbol{z})=\prod_{i=1}^{n} \pi_{i}\left(z_{i}\right)
$$

Let the relationship between the vector $Q$ and the observable $u$ given by the forward model $G$ be

$$
\boldsymbol{u}=G(\boldsymbol{q}), G: R^{k} \rightarrow R^{m}
$$

where $u \in R^{m}$ is a vector gathering the response quantities. Here, it is assumed that there exists a deterministic solver (e.g., an FE model code) that takes as input a given set of input parameters $q$ and provides a unique response vector $u$. Typically, the operator $G$ is not given explicitly, but defined by numerically solving some partial differential equation; nevertheless, here, we write this operator in an abstract way.

Since measurement errors are unavoidable in practice, observable data ym may not match the true value of the response $u$; thus, assuming additional observational errors $\varepsilon$, the measurement data take the form

$$
y_{m}=\boldsymbol{u}+\varepsilon=G\left(\boldsymbol{q}_{\text {true }}\right)+\varepsilon,
$$


where $\varepsilon \in R^{m}$ is one realization of a random vector $E: \Omega \rightarrow R^{m}$ modelling the measurement error. Here, we assume $\boldsymbol{E}$ to be some mutually independent Gaussian random variables with joint probability density function (pdf):

$$
\pi_{E}(\varepsilon)=\prod_{i=1}^{m} \pi_{\varepsilon_{i}}\left(\varepsilon_{i}\right)
$$

The Bayesian approach seeks to estimate the updated density of the random vector $Q$ given a set of observations $\boldsymbol{y}_{m}$. The Bayes rule takes the form

$$
\pi_{Q \mid y_{m}}(\boldsymbol{q})=\frac{\pi_{y_{m} \mid \boldsymbol{q}}\left(\boldsymbol{y}_{\boldsymbol{m}} \mid \boldsymbol{q}\right) \pi_{Q}(\boldsymbol{q})}{\int \pi_{y_{m} \mid \boldsymbol{q}}\left(\boldsymbol{y}_{\boldsymbol{m}} \mid \boldsymbol{q}\right) \pi_{Q}(\boldsymbol{q}) d \boldsymbol{q}}
$$

where $\pi_{Q}(\boldsymbol{q})$ is the prior probability density of $Q ; \pi_{y_{m} \mid q}\left(\boldsymbol{y}_{\boldsymbol{m}} \mid \boldsymbol{q}\right)$ is the likelihood function; and $\pi_{Q \mid y_{m}}(q)$ is the density of $Q$ conditional on the data $y_{m^{\prime}}$ or, in other words, the posterior probability density of $Q$. In this case, the likelihood reads

$$
L(\boldsymbol{q})=\pi_{\boldsymbol{y}_{\boldsymbol{m}} \mid \boldsymbol{q}}\left(\boldsymbol{y}_{\boldsymbol{m}} \mid \boldsymbol{q}\right)=\prod_{i=1}^{m} \pi_{\varepsilon_{i}}\left(y_{m, i}-u_{i}\right)
$$

It must be recalled that, in most cases, the likelihood and thus the posterior distribution cannot be analytically derived in a closed form.

\subsection{Numerical Approach to the Bayesian Updating}

\subsubsection{Surrogate Modelling: The Polynomial Chaos Expansion}

Let us consider an engineering system. Performing uncertainty quantification analysis, as well as solving Bayesian inverse problems for such a system, may require running the forward model $G$ many times with a high computational cost. In order to speed up the computations, a proxy model for the predicted measurable $u$ replacing the $G$ map can be extremely helpful. Here, the PCE surrogate model is adopted. For an extensive review of this topic, please refer to [51].

Without going into mathematical details, if $\boldsymbol{U}=G(\boldsymbol{Q})$ has a finite variance, the $\boldsymbol{U}_{h}$ approximation of the response reads

$$
\boldsymbol{U}=G\left(F^{-1}(\boldsymbol{Z})\right) \approx \boldsymbol{U}_{h}=\sum_{i} \hat{\boldsymbol{u}}_{i} \Phi_{i}(\mathbf{Z})
$$

where $\Phi_{i}(\boldsymbol{Z})=\phi_{0}, \ldots, \phi_{n}$ are the PCE basis functions, some orthogonal multivariate polynomials of total degree less than or equal to $N$, and $\hat{\boldsymbol{u}}_{i}$ are the coefficients of the PCE. As $Z$ is a Gaussian vector, the corresponding orthogonal polynomials with respect to the Gaussian measure are the Hermite polynomials. The computation of the coefficients can be carried out with different methods, such as interpolation, regression, or pseudo-spectral projection. For further details about this topic, please refer to [7,51]. When the PCE of a given forward model is available, one has, in fact, an analytical representation of $\boldsymbol{u}$ in terms of $z$, with the advantage that, for any realization of the random parameter $Q$, the response $u$ can be easily evaluated by first mapping $q$ to $z$ and then evaluating the PCE, with not much computational expense. This is crucial when the deterministic solver is computationally demanding. Furthermore, statistical information can be also analytically computed in a straightforward and efficient manner. Similarly, the parameter $Q$ and the measurement error $\boldsymbol{E}$ can also be represented with a PCE expansion:

$$
\begin{gathered}
Q_{h}=F^{-1}(\boldsymbol{Z})=\sum_{i} \hat{\boldsymbol{q}}_{i} \Phi_{i}(\boldsymbol{Z}) \\
\boldsymbol{E}=\sum_{i} \hat{\boldsymbol{e}}_{i} \Phi_{i}(\boldsymbol{X}),
\end{gathered}
$$


where $\Phi_{i}(\boldsymbol{Z})$ and $\Phi_{i}(\boldsymbol{X})$ are the PCE basis functions, $\hat{\boldsymbol{q}}_{i}$ and $\hat{\boldsymbol{e}}_{i}$ are the corresponding PCE coefficients and $X$ is a set of standardized Gaussian random variables. Then, assuming an additive error (Equation (3)), the measurement model $Y$ can be written with the PCE:

$$
Y_{h}=U_{h}+E=\sum_{\mathrm{i}} \hat{u}_{i} \Phi_{i}(Z)+\sum_{\mathrm{j}} \hat{\boldsymbol{e}}_{j} \Phi_{j}(X)
$$

The data $y_{m}$ that will be measured represent a sample of the $Y$ measurement model. To simplify the computations, the two PCE representations in Equation (10) can be combined, obtaining a single PCE:

$$
Y_{h}=\sum_{j} \hat{y}_{j} \Phi_{c, j}(Z, X),
$$

where $\Phi_{c, j}$ is the same PCE basis adopted for $\boldsymbol{U}_{h}$, but further extended with the basis function of $E$ [24].

\subsubsection{The Markov Chain Monte Carlo (MCMC) Method}

As mentioned before, in most cases, the posterior distribution $\pi_{Q \mid y_{m}}(\boldsymbol{q})$ cannot be derived in a closed form, but it can be estimated by sampling techniques. One of the most used method is the MCMC. The algorithm of the MCMC samples from the posterior distribution, with the help of a random walk, constructs a Markov chain that has the desired pdf as its equilibrium distribution [52]. This approach is not only very general and intuitive, but also easy to compute. Nevertheless, the MCMC algorithm is a very demanding procedure; in fact, the system response must be individually evaluated for each new proposed sample.

A random walk starts at a point of the parameter space $q^{(j)}$ of the $Q$, e.g., at the mean value of the a priori distribution. Considering $j=0$, the sample $\boldsymbol{q}^{(j)}$ is plugged into the forward model $G$. Once the response $\boldsymbol{u}^{(j)}=G\left(\boldsymbol{q}^{(j)}\right)$ is computed, the likelihood $L\left(\boldsymbol{q}^{(j)}\right)$ from Equation (6) and the prior probability $\pi\left(\boldsymbol{q}^{(j)}\right)$ can be easily calculated. The normalization constants of the denominator in Equation (5), which are hard to compute, do not have to be evaluated since the acceptance probability of the random walk only involves the computation of the numerator in Equation (5). Then, a proposal is made to randomly change the value of the parameters to $\boldsymbol{q}^{(j+1)}$ by sampling from the so-called proposal density. At this new value, the numerator of Equation (5) is recomputed. If the numerator in the new point $\boldsymbol{q}^{(j+1)}$ is higher than the one at the actual point $\boldsymbol{q}^{(j)}$, the new point is accepted as a sample point. If it is lower, the closer the numerator (and thus the posterior probability) at this new sample point is to the numerator at the previous point, the higher the probability is of accepting this new value of the parameters. When the proposal distribution is symmetric, the probability of acceptance of each step $j+1$ is computed from the ratio of the posterior probabilities, which is

$$
r=\min \left\{1, \frac{L\left(\boldsymbol{q}^{(j+1)}\right) \pi_{\boldsymbol{Q}}\left(\boldsymbol{q}^{(j+1)}\right)}{L\left(\boldsymbol{q}^{(j)}\right) \pi_{\boldsymbol{Q}}\left(\boldsymbol{q}^{(j)}\right)}\right\} .
$$

When the step is not accepted, then the $(j+1)$ - th sample point is the same as the previous $j$ - th sample point of $Q$. The process is repeated till the equilibrium distribution is reached. The speed of the convergence may significantly depend on the initial step. Because the beginning of the chain is still influenced by the starting point, it is common to exclude a few starting samples from the chain, the so-called "burn in period" [24]. In order to improve the efficiency of the MCMC method, the introduced PCE surrogate model can be used, instead of using the computationally expensive FE solver, for all the samples [53].

Here, we implemented a parallelized multiple chain version of the algorithm that could further speed up the computations. The algorithm is described in Appendix A. 


\subsubsection{Ensemble Kalman Filter and the Sample Free Kalman Filter}

The Kalman Filter (KF) was initially proposed as a method for sequential state estimation for incompletely observable, linear discrete-time dynamics [45]. The KF scheme can be also applied to solve the inverse problem [54]. As the KF assumes that the parameters to be updated are Gaussian, we update the $Z$ parameters instead of $Q$ and then map it back to $\boldsymbol{Q}=F^{-1}(\boldsymbol{Z})$.

The updated parameter $Q^{\prime}$ is determined as a combination of the prior parameter $Q$ and the measurement $y_{m}$ from

$$
Q^{\prime}=Q+K\left(y_{m}-Y\right)
$$

where $K$ is the Kalman gain:

$$
K=\operatorname{Cov}(\boldsymbol{Q}, \boldsymbol{U})[\operatorname{Cov}(\boldsymbol{U})+\operatorname{Cov}(\boldsymbol{E})]^{-1} .
$$

The main assumption of Kalman filtering is that the map from parameter $Q$ to the measurable response $\boldsymbol{U}$ can be written in a linear form. To overcome this limitation, various approximations have been developed. Among others, the Ensemble Kalman Filter (EnKF) keeps the form of the filter given in (14), but computes the covariances by MC samples of $Q, U$, and error $E[30,55]$. To avoid the sampling procedure required by the EnKF, one may resort again to a functional approximation of the random variables; in this light, the linear Bayesian procedure is reduced to a simple algebraic method [51].

In the sample free Kalman filter (PCE-KF), the random input $Q$, the predicted system response $U$ and the measurement model $Y$ can be all represented in a polynomial chaos expansion form, using the approximations in Equations (8)-(10). It is then possible to approximate Equation (13) by

$$
Q_{h}{ }^{\prime}=Q_{h}+K\left(y_{m}-Y_{h}\right) .
$$

where $K$ is the Kalman gain that is calculated according to Equation (14) in an algebraic way, evaluating the covariance matrices directly from the PCE coefficients $\hat{\boldsymbol{q}}_{i}, \hat{\boldsymbol{u}}_{i}$ and $\hat{\boldsymbol{e}}_{i}$. It is important to notice that each new measurement enlarges the space we project onto $[24,56]$; thus, as the number of measurements increases, the number of PCE terms involved in the expansion grows and, consequently, so does the computational cost of the method. $Q_{h}{ }^{\prime}$ is described in the combined basis; therefore, before using Equation (15), $Q_{h}$ and the deterministic values $y_{m}$ should be described in terms of this combined basis $\Phi_{c, j}$. More details about the implementation of the algorithm can be found in [24].

\subsubsection{Method Based on the Minimum Mean Squared Error Estimator}

The advantage of the Kalman Filter when compared with the brute force MCMC is that it is computationally much more efficient, provided that a PCE surrogate model is available providing a closed form of the posterior. With such a closed form, a sequential update becomes possible, so a new update can be computed once new information about the response arrives. To obtain the posterior, the Kalman Filter applies a linear estimator, but the limitation of linearity can be removed by applying a Minimum Mean Square Estimator (MMSE).

Recalling that $Q: \Omega \rightarrow R^{n k}$ is the vector of input random parameters and that $y_{m} \in R^{m}$ is the measurement of the system output, an estimator $\varphi: R^{m} \rightarrow R^{k}$ is any function of the measurement $y_{m}$. Instead of the Bayesian posterior, we formulate the conditional expectation $[48,49]$ as

$$
\hat{\varphi}=\mathbb{E}[\boldsymbol{Q} \mid \boldsymbol{Y}],
$$

where the estimator $\hat{\varphi}$ minimizes the mean square error,

$$
e_{M S E}^{2}=\mathbb{E}\left[(\boldsymbol{Q}-\varphi(\boldsymbol{Y}))^{2}\right]
$$


of $Q$ given the measurement model $\boldsymbol{Y}$ and provides a numerical framework for non-linear filtering [57]. In order to carry out the minimization, $\varphi$ is defined over a finite dimensional function space $\mathcal{V}_{\varphi}$. It is a space spanned by some basis functions $\Psi_{i}$, e.g., some multivariate fixed polynomials. If we restrain the minimization to such subspace, the estimator $\varphi$ can be written as linear combination of these basis functions up to degree $P$ :

$$
\varphi y_{m} \longmapsto \sum_{i} \varphi_{i} \Psi_{i}(Y) .
$$

In this way, the problem of computing the minimizer is reduced to solving a system of linear equations

$$
\mathrm{A} \phi=b .
$$

the unknown coefficients $\varphi_{i}$ in Equation (18) being collected in the vector $\phi$ and

$$
\begin{gathered}
{[\mathrm{A}]_{i j}=\mathbb{E}\left[\Psi_{i}(\boldsymbol{Y}(\boldsymbol{Z}, \boldsymbol{X})) \Psi_{j}(\boldsymbol{Y}(\boldsymbol{Z}, \boldsymbol{X}))\right],} \\
{[b]_{j}=\mathbb{E}\left[\boldsymbol{Q}(\boldsymbol{Z}) \Psi_{j}(\boldsymbol{Y}(\boldsymbol{Z}, \boldsymbol{X}))\right],}
\end{gathered}
$$

being integrals that can be evaluated with the help of numerical integration:

$$
\begin{gathered}
\mathbb{E}\left[\Psi_{i}(\boldsymbol{Y}(\mathbf{Z}, \boldsymbol{X})) \Psi_{j}(\mathbf{Y}(\mathbf{Z}, \boldsymbol{X}))\right] \approx \sum_{k} w_{k} \Psi_{i}(Y(Z, X)) \Psi_{j}(\mathbf{Y}(\mathbf{Z}, \boldsymbol{X})) \\
\mathbb{E}\left[\boldsymbol{Q}(\boldsymbol{Z}) \Psi_{j}(\boldsymbol{Y}(\mathbf{Z}, \boldsymbol{X}))\right] \approx \sum_{k} w_{k} \boldsymbol{Q}(\boldsymbol{Z}) \Psi_{j}(\boldsymbol{Y}(\mathbf{Z}, \boldsymbol{X}))
\end{gathered}
$$

Then, the updated input parameter is described by

$$
Q^{\prime}(Z, Y)=Q(Z)-\varphi(Y(Z, X))+\varphi\left(y_{m}\right)
$$

and the posterior distribution can be estimated directly from samples of the Gaussianindependent random variables $Z$ and $X$. Further details regarding the optimization algorithm are also given in [24,49].

\section{Benchmark Study}

The performance of the methods described in the previous section is investigated with respect to the most relevant issues: the degree of non-linearity of the model, the adopted

\begin{tabular}{|c|c|c|c|c|c|}
\hline Model & $\begin{array}{l}\text { Non-Linearity } \\
\text { of the Model }\end{array}$ & $\begin{array}{c}\text { Magnitude of } \\
\text { Prior Uncertainty }\end{array}$ & $\begin{array}{c}\text { Magnitude of } \\
\text { Measurement Error }\end{array}$ & $\begin{array}{c}\text { Number of } \\
\text { Measurements }\end{array}$ & $\begin{array}{c}\text { Number of Random } \\
\text { Variables }\end{array}$ \\
\hline $\begin{array}{c}\text { Analytical, } \\
\text { abstract models }\end{array}$ & $\checkmark$ & $\checkmark$ & $\checkmark$ & - & - \\
\hline $\begin{array}{l}\text { One-dimensional } \\
\text { static model }\end{array}$ & $\checkmark$ & $\checkmark$ & $\checkmark$ & $\checkmark$ & - \\
\hline $\begin{array}{l}\text { One-dimensional } \\
\text { dynamic model }\end{array}$ & $\checkmark$ & $\checkmark$ & $\checkmark$ & - & $\checkmark$ \\
\hline FE model & $\checkmark$ & $\checkmark$ & $\checkmark$ & $\checkmark$ & $\checkmark$ \\
\hline
\end{tabular}
prior distribution, the measurement error, the information content of the measurements and the number of random variables. The benchmark models introduced in the section "Scope of the Overview" are considered in this study. However, some models are more suitable than others for investigating specific issues; Table 1 shows a comprehensive overview of the aspects investigated through the different models.

Table 1. Overview of the benchmark study: investigated issues and models considered. 
The numerical results of the benchmark study are given in Tables 2-11 in terms of posterior modes and percentage deviation from the assumed true values of the random parameters, while graphical representations are provided in the relevant Figures. The diagrams illustrated in these have a double $y$-axis, with the left axis being used for PDF and the right axis for the model response. Each figure reports:

- The prior PDF, in blue;

- $\quad$ The posterior PDFs obtained via MCMC (cyan), via PCE-KF (green) and via NL-MMSE (red solid line);

- The true value of the parameter $q_{\text {true }}$ (red dashed line);

- The model response $u(q)$, in black;

- The measurement $y_{m}$ from which the update was computed (black dashed line);

- $\quad$ The $\pm 3 \sigma_{\varepsilon}$ region of the measurement (grey area).

The updating algorithms, which were developed from the open source stochastic library called SGLIB [58], are set up in the following way:

- For MCMC, the starting point of the random walk was the mean of the prior distribution and running multiple chains, as discussed in the Appendix; the posterior distribution was described by 50,000 or more samples;

- For the NL-MMSE, a degree $p=10$ was used for the polynomial approximation, on the basis of the updating results obtained with different degrees of approximation.

Table 2. Performance of the different updating procedures: comparison of posterior mode $q_{m}^{\prime}$ and $q_{\text {true }}=u+\sigma=2.5$.

\begin{tabular}{ccccccccc}
\hline \multirow{2}{*}{ Method } & \multicolumn{2}{c}{ Model 1 } & \multicolumn{2}{c}{ Model 2a } & \multicolumn{2}{c}{ Model 2b } & \multicolumn{2}{c}{ Model 3 } \\
\cline { 2 - 9 } & $\boldsymbol{q}_{\boldsymbol{m}}^{\prime}$ & $\boldsymbol{\Delta} \%$ & $\boldsymbol{q}_{\boldsymbol{m}}^{\prime}$ & $\boldsymbol{\Delta} \%$ & $\boldsymbol{q}_{\boldsymbol{m}}^{\prime}$ & $\boldsymbol{\Delta} \%$ & $\boldsymbol{q}_{\boldsymbol{m}}^{\prime}$ & $\boldsymbol{\Delta} \%$ \\
\hline MCMC & 2.454 & 1.84 & 2.503 & 0.12 & 2.495 & 0.20 & 2.497 & 0.12 \\
\hline PCE-KF & 2.466 & 1.36 & 2.494 & 0.24 & 2.457 & 1.72 & 2.498 & 0.08 \\
\hline MMSE & 2.442 & 2.32 & 2.495 & 0.20 & 2.501 & 0.04 & 2.506 & 0.24 \\
\hline
\end{tabular}

Table 3. Performance of the different updating procedures: comparison of posterior mode $q_{m}^{\prime}$ and $q_{\text {true }}=u+3 \sigma=3.5$.

\begin{tabular}{ccccccccc}
\hline \multirow{2}{*}{ Method } & \multicolumn{2}{c}{ Model 1 } & \multicolumn{2}{c}{ Model 2a } & \multicolumn{2}{c}{ Model 2b } & \multicolumn{2}{c}{ Model 3 } \\
\cline { 2 - 9 } & $\boldsymbol{q}_{\boldsymbol{m}}^{\prime}$ & $\boldsymbol{\Delta} \%$ & $\boldsymbol{q}_{\boldsymbol{m}}^{\prime}$ & $\boldsymbol{\Delta} \%$ & $\boldsymbol{q}_{\boldsymbol{m}}^{\prime}$ & $\boldsymbol{\Delta} \%$ & $\boldsymbol{q}_{\boldsymbol{m}}^{\prime}$ & $\boldsymbol{\Delta} \%$ \\
\hline MCMC & 3.235 & 7.57 & 3.457 & 1.23 & 3.480 & 0.57 & 3.484 & 0.46 \\
\hline PCE-KF & 3.244 & 7.31 & 3.761 & 7.46 & 3.799 & 8.54 & 4.496 & 28.46 \\
\hline MMSE & 3.249 & 7.17 & 3.467 & 0.94 & 3.679 & 5.11 & 3.502 & 0.06 \\
\hline
\end{tabular}

Table 4. Performance of the different updating procedures: comparison of posterior mode $E J_{m}^{\prime}$ and $E J_{\text {true }}=m+s=1.2$.

\begin{tabular}{ccccccc}
\hline \multirow{2}{*}{ Method } & \multicolumn{2}{c}{$\mathbf{1}$ Measurement } & \multicolumn{2}{c}{ 2 Measurements } & \multicolumn{2}{c}{ 3 Measurements } \\
\cline { 2 - 7 } & $\boldsymbol{E} \boldsymbol{J}_{\boldsymbol{m}}^{\prime}$ & $\boldsymbol{\Delta} \boldsymbol{\%}$ & $\boldsymbol{E} \boldsymbol{J}_{\boldsymbol{m}}^{\prime}$ & & $\boldsymbol{E} \boldsymbol{J}_{\boldsymbol{m}}^{\prime}$ & $\boldsymbol{\Delta} \boldsymbol{0}$ \\
\hline MCMC & 1.187 & 1.08 & 1.193 & 0.58 & 1.201 & 0.33 \\
\hline PCE-KF & 1.175 & 2.08 & 1.174 & 2.17 & 1.122 & 1.92 \\
\hline MMSE & 1.181 & 1.58 & 1.19 & 0.83 & 1.195 & 0.5 \\
\hline
\end{tabular}


Table 5. Performance of the different updating procedures: comparison of posterior mode $E J_{m}^{\prime}$ and $E J_{\text {true }}=m+3 s=1.6$.

\begin{tabular}{ccccccc}
\hline \multirow{2}{*}{ Method } & \multicolumn{2}{c}{$\mathbf{1}$ Measurement } & \multicolumn{2}{c}{ 2 Measurements } & \multicolumn{2}{c}{ 3 Measurements } \\
\cline { 2 - 7 } & $\boldsymbol{E} \boldsymbol{J}_{\boldsymbol{m}}^{\prime}$ & $\boldsymbol{\Delta} \%$ & $\boldsymbol{E} \boldsymbol{J}_{\boldsymbol{m}}^{\prime}$ & & $\boldsymbol{E} \boldsymbol{J}_{\boldsymbol{m}}^{\prime}$ & $\boldsymbol{\Delta} \%$ \\
\hline MCMC & 1.558 & 2.63 & 1.578 & 1.38 & 1.582 & 1.13 \\
\hline PCE-KF & 1.424 & 11 & 1.427 & 10.81 & 1.428 & 10.75 \\
\hline MMSE & 1.554 & 2.88 & 1.576 & 1.5 & 1.584 & 1 \\
\hline
\end{tabular}

Table 6. Performance of the different updating procedures after mapping the response: comparison of posterior mode $E J_{m}^{\prime}$ and $E J_{\text {true }}=m+s=1.2$.

\begin{tabular}{ccccccc}
\hline \multirow{2}{*}{ Method } & \multicolumn{2}{c}{$\mathbf{1}$ Measurement } & \multicolumn{2}{c}{ 2 Measurements } & \multicolumn{2}{c}{ 3 Measurements } \\
\cline { 2 - 7 } & $\boldsymbol{E} \boldsymbol{J}_{\boldsymbol{m}}^{\prime}$ & $\boldsymbol{\Delta} \%$ & $\boldsymbol{E} \boldsymbol{J}_{\boldsymbol{m}}^{\prime}$ & & $\boldsymbol{E} \boldsymbol{J}_{\boldsymbol{m}}^{\prime}$ & $\boldsymbol{\Delta} \%$ \\
\hline MCMC & 1.186 & 1.17 & 1.188 & 1 & 1.195 & 0.42 \\
\hline PCE-KF & 1.175 & 2.08 & 1.19 & 0.83 & 1.191 & 0.75 \\
\hline MMSE & 1.19 & 0.83 & 1.192 & 0.67 & 1.195 & 0.42 \\
\hline
\end{tabular}

Table 7. Performance of the different updating procedures after mapping the response: comparison of posterior mode $E J_{m}^{\prime}$ and $E J_{\text {true }}=m+3 s=1.6$.

\begin{tabular}{ccccccc}
\hline \multirow{2}{*}{ Method } & \multicolumn{2}{c}{$\mathbf{1}$ Measurement } & \multicolumn{2}{c}{ 2 Measurements } & \multicolumn{2}{c}{ 3 Measurements } \\
\cline { 2 - 7 } & $\boldsymbol{E} \boldsymbol{J}_{\boldsymbol{m}}^{\prime}$ & $\boldsymbol{\Delta} \%$ & $\boldsymbol{E} \boldsymbol{J}_{\boldsymbol{m}}^{\prime}$ & & $\boldsymbol{E} \boldsymbol{J}_{\boldsymbol{m}}^{\prime}$ & $\boldsymbol{\Delta} \%$ \\
\hline MCMC & 1.546 & 3.38 & 1.567 & 2.06 & 1.593 & 0.44 \\
\hline PCE-KF & 1.630 & 1.88 & 1.691 & 5.69 & 1.72 & 7.5 \\
\hline MMSE & 1.559 & 2.56 & 1.566 & 2.13 & 1.594 & 0.38 \\
\hline
\end{tabular}

Table 8. Comparison of posterior mode $E J_{m}^{\prime}$ and $E J_{\text {true }}$ according to different updating procedures.

\begin{tabular}{ccccccc}
\hline \multirow{2}{*}{ Method } & \multicolumn{2}{c}{$E J_{\text {true }}=\overline{E J}+s_{E J}=1.2$} & \multicolumn{2}{c}{$E J_{\text {true }}=\overline{E J}+2$} & $s_{E J}=1.4$ & \multicolumn{2}{c}{$E J_{\text {true }}=\overline{E J}+3$} & $s_{E J}=1.6$ \\
\cline { 2 - 7 } & $E J_{m}^{\prime}$ & $\boldsymbol{\Delta} \%$ & $E J_{m}^{\prime}$ & $E J_{m}^{\prime}$ & $\Delta \%$ & $\Delta \%$ \\
\hline MCMC & 1.18 & 1.92 & 1.37 & 1.86 & 1.55 & 2.87 \\
\hline PCE-KF & 1.17 & 2.08 & 1.38 & 1.36 & 1.59 & 0.69 \\
\hline MMSE & 1.19 & 0.75 & 1.38 & 1.57 & 1.56 & 2.25 \\
\hline
\end{tabular}

Table 9. Comparison of posterior mode $E_{m}^{\prime}$ and $E_{\text {true }}$ according to different updating procedures by measuring the fundamental natural frequency $f_{1}$.

\begin{tabular}{|c|c|c|c|c|c|c|}
\hline \multirow[t]{2}{*}{ Method } & \multicolumn{2}{|c|}{$E_{\text {true }}=\bar{E}+s_{E}=52$} & \multicolumn{2}{|c|}{$E_{t r u e}=\bar{E}+2 s_{E}=62$} & \multicolumn{2}{|c|}{$E_{\text {true }}=\bar{E}+3 s_{E}=72$} \\
\hline & $E J_{m}^{\prime}$ & $\Delta \%$ & $E J_{m}^{\prime}$ & $E J_{m}^{\prime}$ & $\Delta \%$ & $E J_{m}^{\prime}$ \\
\hline MCMC & 51.45 & 1.05 & 61.15 & 1.36 & 71.28 & 1.01 \\
\hline PCE-KF & 51.58 & 0.81 & 62.83 & 1.35 & 75.43 & 4.77 \\
\hline MMSE & 51.87 & 0.25 & 61.66 & 0.55 & 71.49 & 0.71 \\
\hline
\end{tabular}


Table 10. Comparison of posterior mode $E_{m}^{\prime}$ and $E_{\text {true }}$ according to different updating procedures by measuring the displacement in two different points.

\begin{tabular}{|c|c|c|c|c|c|c|}
\hline \multirow{2}{*}{ Method } & \multicolumn{2}{|c|}{$E_{t r u e}=\bar{E}+s_{E}=52$} & \multicolumn{2}{|c|}{$E_{\text {true }}=\bar{E}+2 s_{E}=62$} & \multicolumn{2}{|c|}{$E_{\text {true }}=\bar{E}+3 s_{E}=72$} \\
\hline & $E J_{m}^{\prime}$ & $\Delta \%$ & $E J_{m}^{\prime}$ & $E J_{m}^{\prime}$ & $\Delta \%$ & $E J_{m}^{\prime}$ \\
\hline MCMC & 51.58 & 0.81 & 60.65 & 2.18 & 69.51 & 3.46 \\
\hline PCE-KF & 50.36 & 3.15 & 56.58 & 8.75 & 61.83 & 14.13 \\
\hline MMSE & 51.54 & 0.89 & 60.86 & 1.84 & 69.81 & 3.05 \\
\hline
\end{tabular}

Table 11. Comparison of posterior mode $E_{m}^{\prime}$ and $E_{\text {true }}$ according to different updating procedures (after mapping) by measuring the displacement in two different points.

\begin{tabular}{|c|c|c|c|c|c|c|}
\hline \multirow{2}{*}{ Method } & \multicolumn{2}{|c|}{$E_{\text {true }}=\bar{E}+s_{E}=52$} & \multicolumn{2}{|c|}{$E_{\text {true }}=\bar{E}+2 s_{E}=62$} & \multicolumn{2}{|c|}{$E_{\text {true }}=\bar{E}+3 s_{E}=72$} \\
\hline & $E J_{m}^{\prime}$ & $\Delta \%$ & $E J_{m}^{\prime}$ & $E J_{m}^{\prime}$ & $\Delta \%$ & $E J_{m}^{\prime}$ \\
\hline MCMC & 51.70 & 0.57 & 60.73 & 2.04 & 69.26 & 3.81 \\
\hline PCE-KF & 51.45 & 1.05 & 62.33 & 0.53 & 74.55 & 3.54 \\
\hline MMSE & 51.48 & 1.00 & 60.64 & 2.19 & 69.23 & 3.85 \\
\hline
\end{tabular}

\subsection{Analytical Abstract Models}

The stochastic inverse methods previously described are first tested on four analytical abstract models, characterized by an increasing degree of non-linearity:

- $\quad$ Model 1: $u(q)=1+\alpha q, \alpha=1$;

- $\quad$ Model 2a: $u(q)=1+q^{2}$;

- $\quad$ Model 2b: $u(q)=8-7 q+2 q^{2}$;

- Model 3: $u(q)=1+0.3 q^{4}$.

It is important to notice that, since these models are explicit, the model response is already a polynomial; a response surface approximating the system response can be still defined by means of the PCE theory. Nevertheless, when working with real-life problems, unfortunately, the surrogate modelling can introduce additional errors in the inversion.

Assuming the input parameter $Q$ is normally distributed $N(\mu=2, \sigma=0.5)$, synthetic measurements are considered and are obtained by setting the true value of the input random variable $q_{\text {true }}$ to $\mu+\sigma$ and $\mu+3 \sigma$. The measurement data $y_{m}$ were generated according to Equation (3), where $\varepsilon$ was sampled from the distribution of the error which was set to be a normal distribution with zero mean and initial standard deviation $\sigma_{\varepsilon}=u\left(q_{\text {true }}\right) / 20$.

In Figure 1, the results of the update are shown for $q_{\text {true }}=\mu+\sigma$ and, in Figure 2, those for $q_{\text {true }}=\mu+3 \sigma$, while, in Tables 2 and 3, they are summarized in terms of the posterior mode $q_{m}^{\prime}$, the true value of the parameter $q_{\text {true }}$ and the percentage deviation from the true value, $\Delta \%$. 


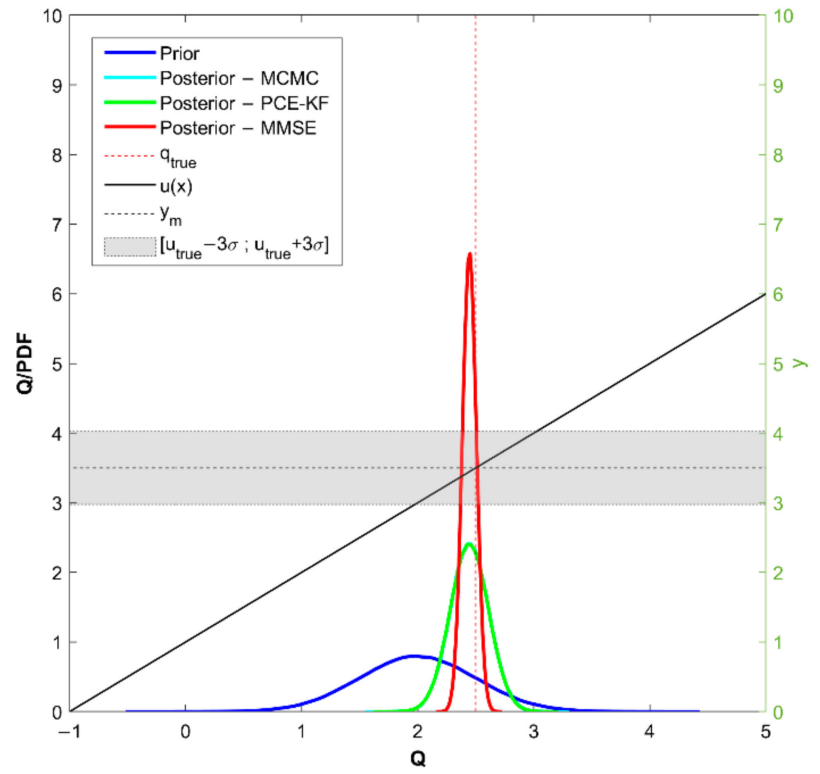

(a)

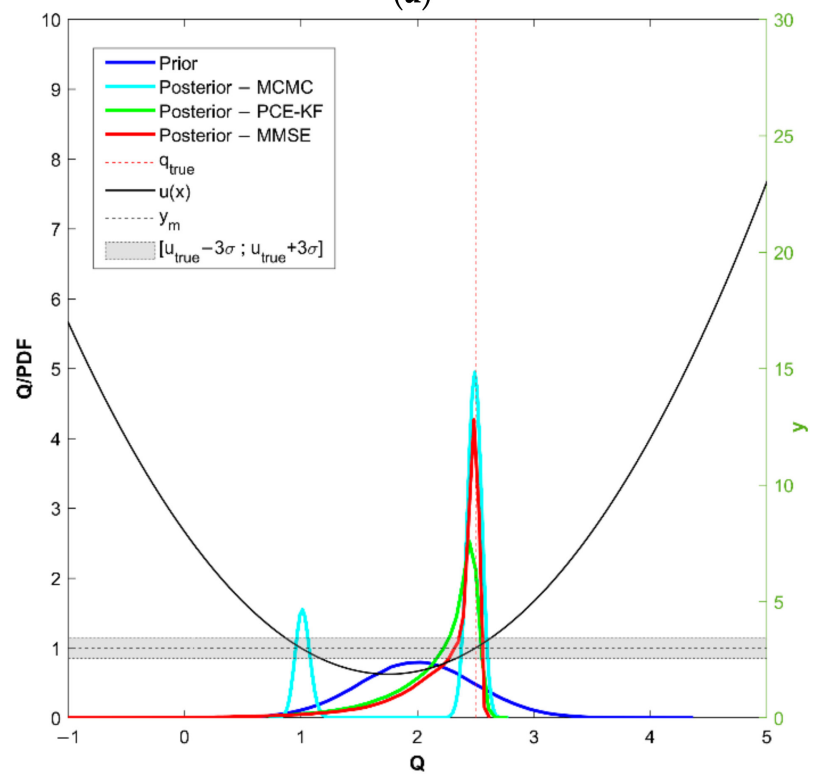

(c)

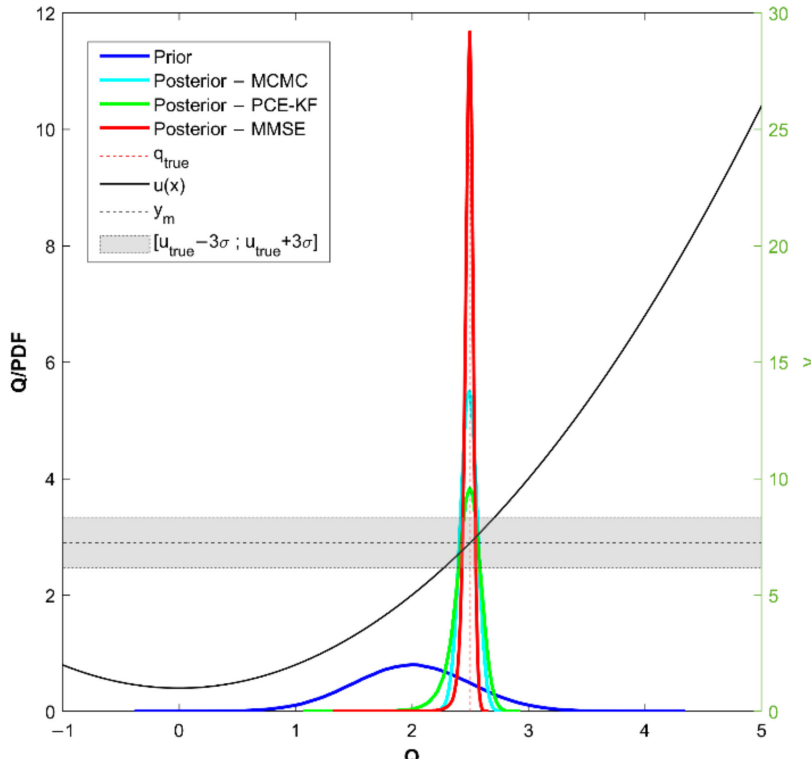

(b)

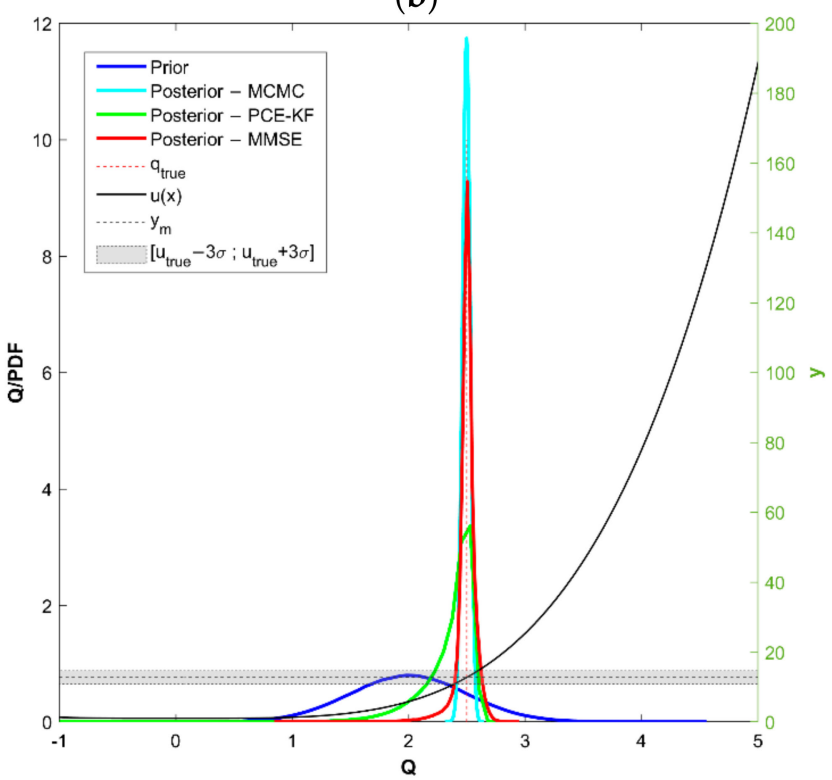

(d)

Figure 1. Comparison of different updating methods considering $q_{\text {true }}=\mu+\sigma$ : (a) Model 1, (b) Model 2a, (c) Model 2b and (d) Model 3. In each figure, the following are reported: the prior PDF (in blue); the posterior PDFs obtained via MCMC (in cyan), via PCE-KF (in green) and via NL-MMSE (red solid line); the true value of the parameter $q_{\text {true }}$ (red dashed line); the model response $u(q)$ (black solid line); the measurement $y_{m}$ (black dashed line); the $\pm 3 \sigma_{\varepsilon}$ region of the measurement (grey area). 


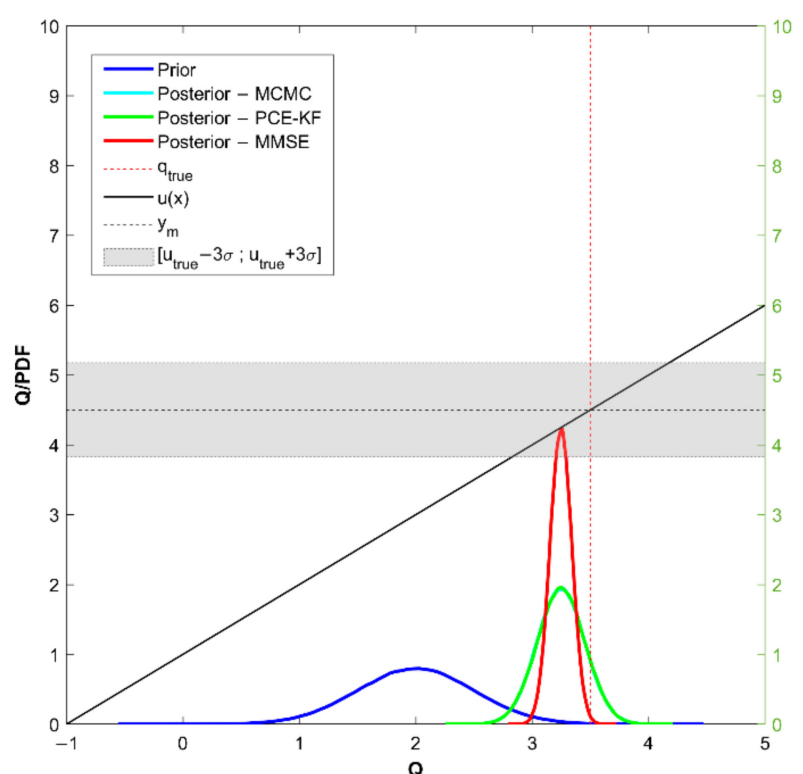

(a)

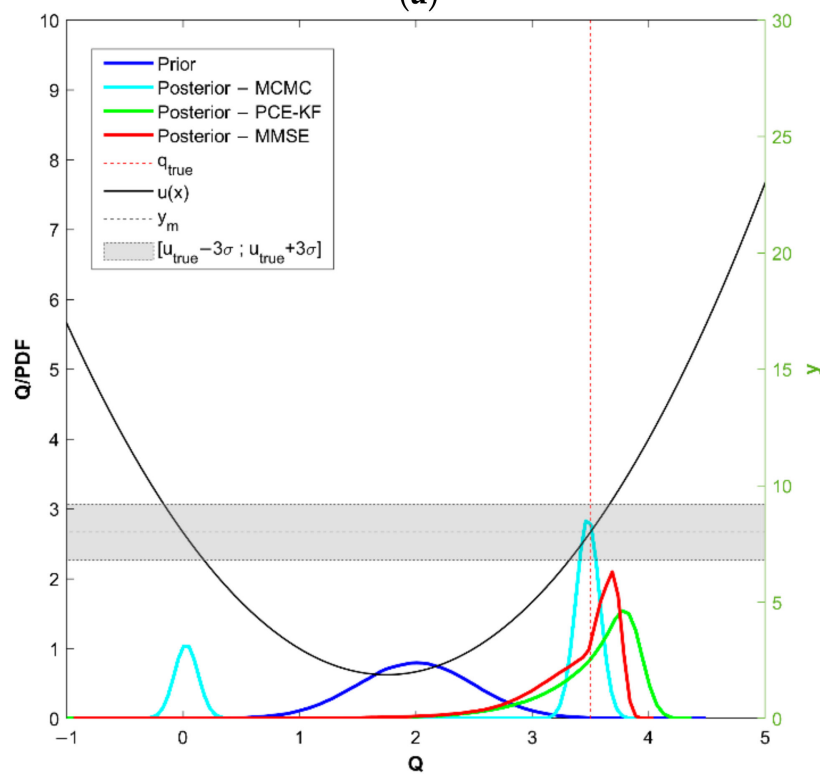

(c)

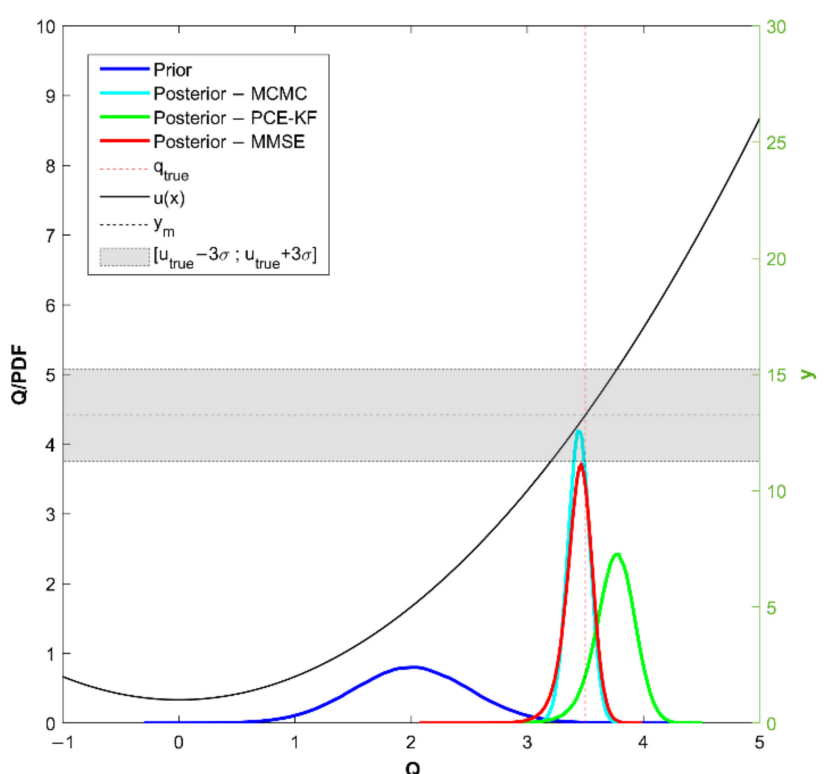

(b)

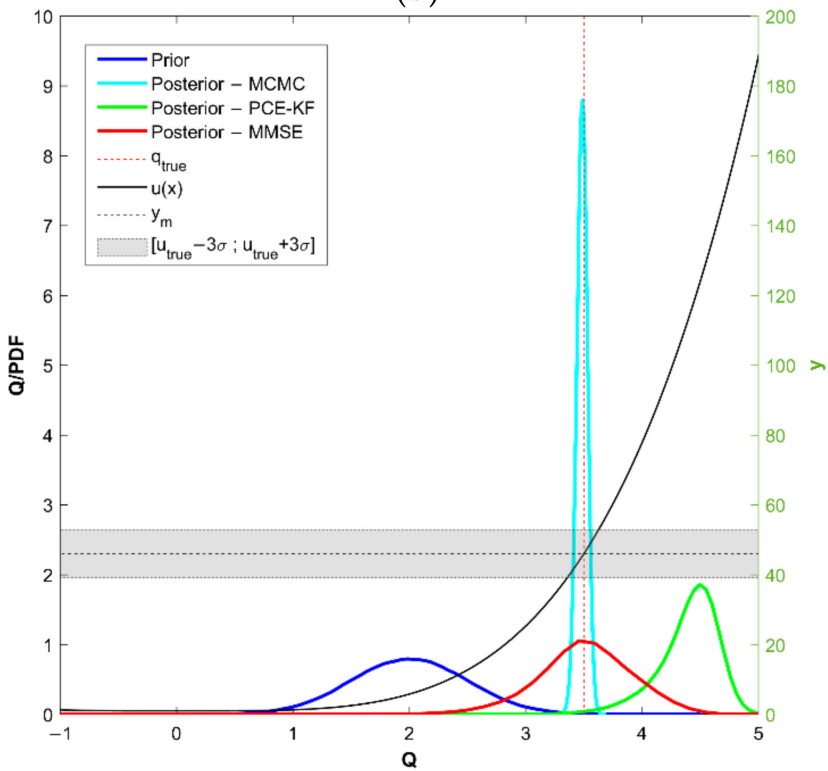

(d)

Figure 2. Comparison of different updating methods considering $q_{\text {true }}=\mu+3 \sigma$ : (a) Model 1, (b) Model 2a, (c) Model 2b and (d) Model 3. In each figure, the following are reported: the prior PDF (in blue); the posterior PDFs obtained via MCMC (in cyan), via PCE-KF (in green) and via NL-MMSE (red solid line); the true value of the parameter $q_{\text {true }}$ (red dashed line); the model response $u(q)$ (black solid line); the measurement $y_{m}$ (black dashed line); the $\pm 3 \sigma_{\varepsilon}$ region of the measurement (grey area).

Considering Model 3 and $q_{\text {true }}=\mu+\sigma$, we notice that the posterior distribution obtained with the PCE-KF, which is a linear filter, succeeds on recovering the Bayesian posterior that we obtain by the MCMC. However, in case of an inadequate level of knowledge, represented by a true value rather distant from the prior assumption $q_{\text {true }}=\mu+3 \sigma$, the PCE-KF is not applicable because of its high deviation. The KF tries to linearize the model using the high probability region of the prior. Due to the flat slope here, the KF overshoots the posterior. Increasing the degree of the estimator by MMSE improves the results. It must be stressed that, for some problems, the polynomial approximation of the estimator is not suitable for inverting highly non-linear models, according to the limitations pointed out in [59]. 
As already explained, the inverse problem is ill-posed and this issue is particularly relevant in the case of Model $2 \mathrm{~b}$, since, for each possible output of the model, two values of the input can be assigned. Assuming that, in the Bayesian framework, the problem becomes well posed at the price of "only" obtaining probability distributions on the possible values of $q$ [7], we speculate that the posterior distribution should be bimodal. However, the only method among those analyzed, which is capable of delivering the bimodal distribution, is the MCMC. Nevertheless, the mean of the posterior recovered by the MMSE approximates the mean of the Bayesian posterior sufficiently enough.

We study how the sensitivity of the measured response to variation in the input parameter influences the Bayesian posterior. With this aim, we consider the linear model (Model 1) with different slopes $\alpha$ and we compute the deviation $(\Delta \%)$ between the posterior mode $q_{m}^{\prime}$ and the true value $q_{\text {true }}$. Figure 3 clearly shows that, as the slope $\alpha$ increases, the mode of the posterior becomes closer to $q_{\text {true }}$. To analyze the effect of changing the magnitude of the measurement uncertainty, three different values of the standard deviation of the measurement error $\sigma_{\varepsilon}\left(\sigma_{\varepsilon}=0.2,0.4\right.$, and 0.6) were considered for each slope. As we put less trust in the measurement characterized by a higher value of $\sigma_{\varepsilon}$, the Bayesian update puts more weight on the prior knowledge; as a consequence, the posterior mode becomes more distant from the true value. In Figure 3, the deviations are shown for all three methods. Due to the linearity of the model, the three methods should theoretically provide the same result; therefore, the deviations essentially depend on numerical errors.

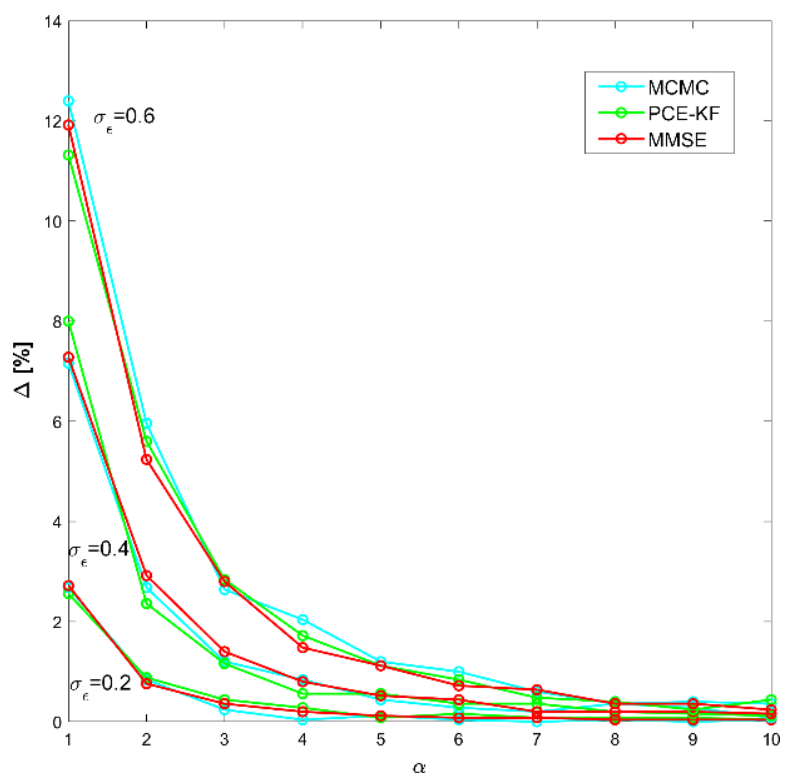

Figure 3. Percentage deviation $\Delta \%$ in the updating plotted as a function of the slope $\alpha$ of the linear model (Model 1) and the measurement error $\sigma_{\mathcal{\varepsilon}}\left(q_{\text {true }}=\mu+\sigma\right)$. Results are compared for the MCMC method (in cyan), the PCE-KF method (in green) and the NL-MMSE method (in red).

Now, we analyze the error update as a function of the measurement error in Figure 4. It must be noticed that the $\Delta \%$ shows a higher dependency in the case of the linear model than in the non-linear models. This is motivated by the fact that the model outputs of the non-linear model are more sensitive to variations in $q$ than the linear model. 


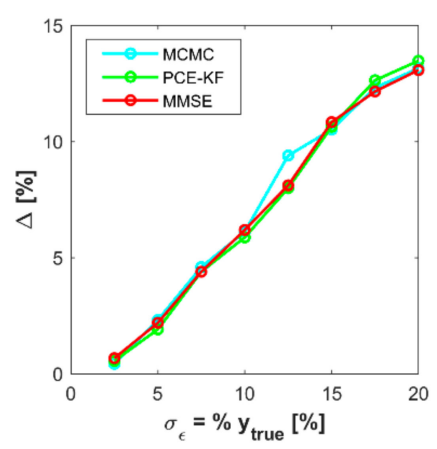

(a)

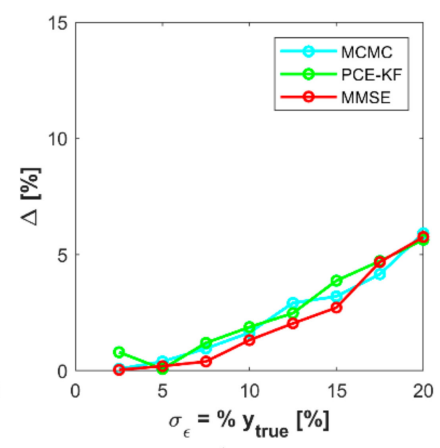

(b)

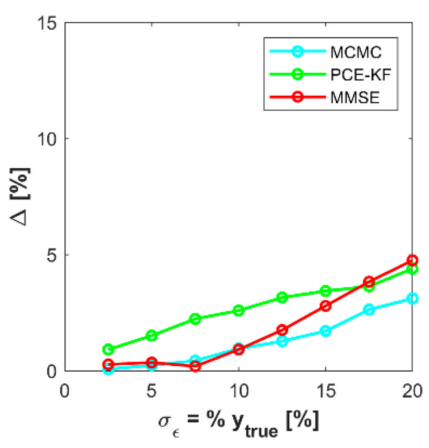

(c)

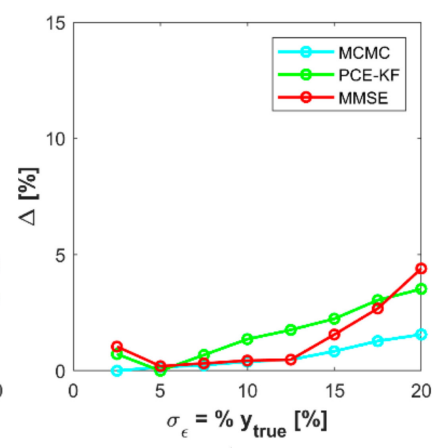

(d)

Figure 4. Percentage deviation $\Delta \%$ in the updating according to different methods, variation with measurement error $\sigma_{\varepsilon}$ considering $q_{\text {true }}=\mu+\sigma$ : (a) Model 1, (b) Model 2a, (c) Model 2b and (d) Model 3. Results are compared for the MCMC method (in cyan), the PCE-KF method (in green) and the NL-MMSE method (in red).

Therefore, in terms of identification problems, special attention should be devoted not only to the global degree of non-linearity of the system response, but also to the local evaluation of first derivatives in the measurement region. Higher derivatives imply a higher sensitivity of the response to the parameter variation in the investigated region [60], thus making parameter identification possible. This should be considered when designing the experiment, e.g., the location of the sensors. In fact, to calibrate the input parameters, outputs should be selected that privilege measurements that are more sensitive to variations in the input parameters themselves.

\subsection{One-Dimensional Static Problem}

Taking inspiration from [61], we consider a one-dimensional static problem, namely, the Euler-Bernoulli cantilever beam shown in Figure 5. The beam, whose length is $L$, is subjected to a deterministic static-distributed load $q$.

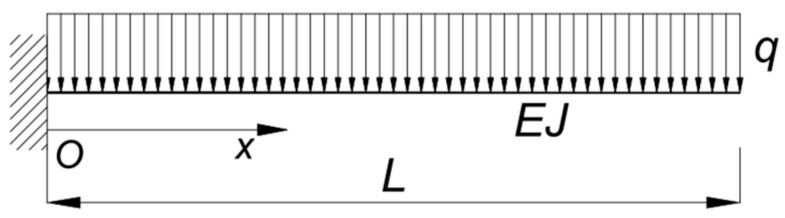

Figure 5. Cantilever beam subjected to uniformly distributed transverse load.

By solving the Euler-Bernoulli equation, we trivially obtain the well-known analytical expression for the deflection $w(x)$ :

$$
w(x)=\frac{q}{24 E J}\left(x^{4}-4 L x^{3}+6 x^{2} L^{2}\right) .
$$

Let us assume from our engineering expertise that the bending rigidity $E J$ has a lognormal prior distribution with mean $m=1$ and standard deviation $s=0.2$. Our reference random variable $Z$ is a standardized Gaussian variable and the map from the reference random variable to $E J$ is

$$
E J=e^{\mathrm{Z} \sigma+\mu},
$$

where $\mu$ and $\sigma$ are the parameters of the lognormal distribution, given by

$$
\mu=\ln \left(\frac{m^{2}}{\sqrt{m^{2}+s^{2}}}\right) \text { and } \sigma^{2}=\ln \left(1+\frac{s^{2}}{m^{2}}\right)
$$


The updating is performed considering:

- One measurement of the deflection at the end of the cantilever $(x=L)$;

- Two measurements of the deflection, at $x=L / 2$ and $x=L$;

- $\quad$ Three measurements of the deflection, at $x=L / 3, x=2 / 3 L$ and $x=L$.

The synthetic measurements are obtained by setting the true value of the input random variable to $m+s$ and $m+3 s$. The results are reported in Tables 4 and 5 and illustrated, when only one available measurement is hypothesized, in Figure 6.

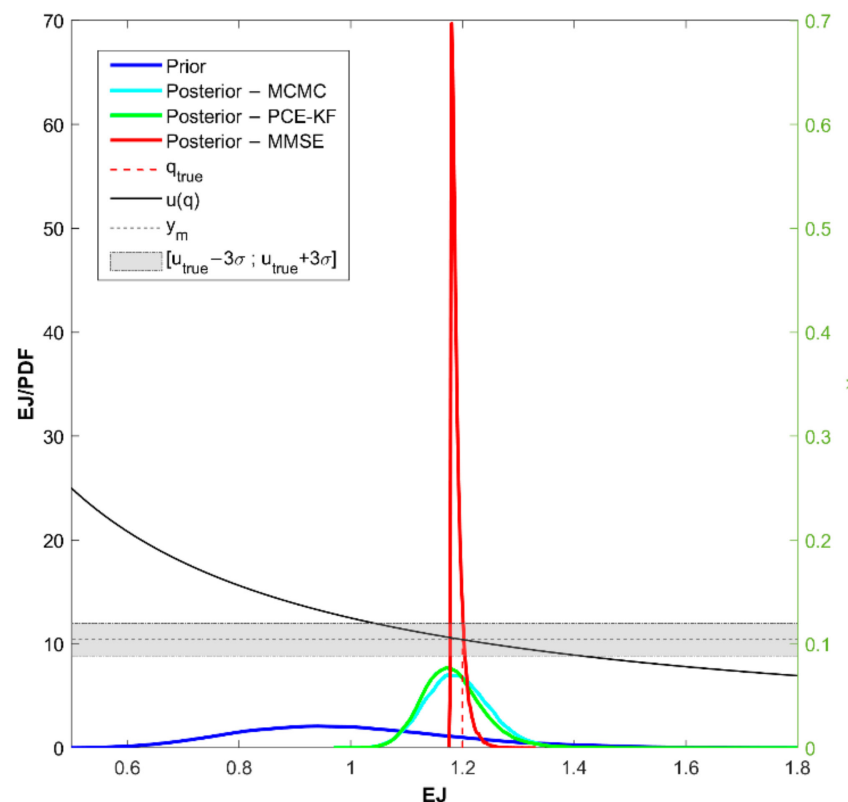

(a)

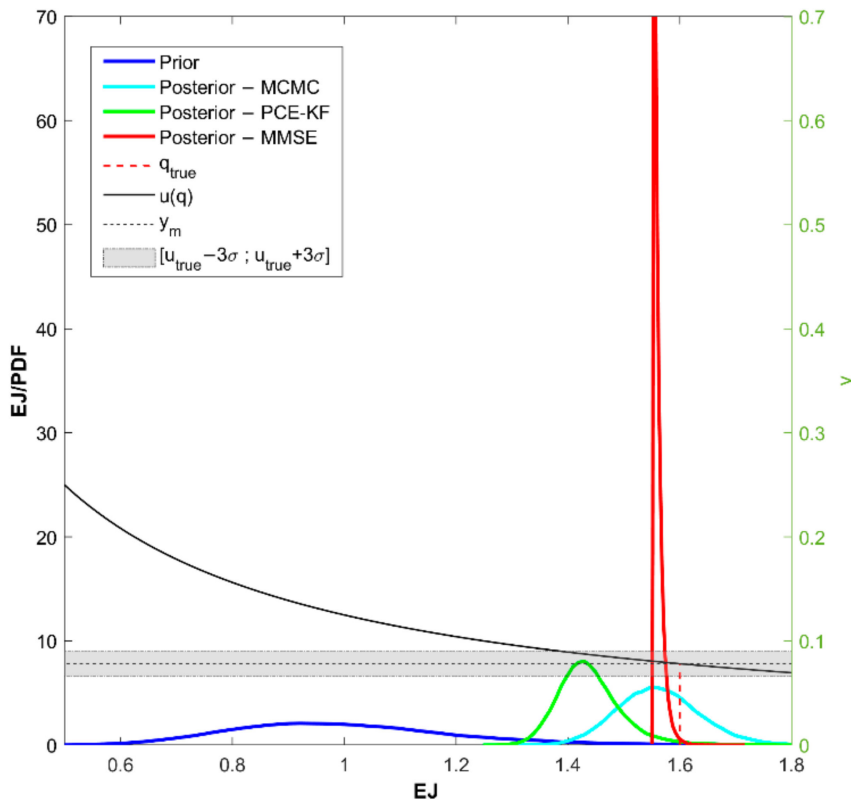

(b)

Figure 6. Comparison of updating procedures-cantilever beam: (a) $E J_{\text {true }}=m+s=1.2$ and (b) $E J_{\text {true }}=m+3 s=1.6$. In each figure, the following are reported: the prior PDF (in blue); the posterior PDFs obtained via MCMC (in cyan), via PCE-KF (in green) and via NL-MMSE (red solid line); the true value of the parameter $q_{\text {true }}$ (red dashed line); the model response $u(q)$ (black solid line); the measurement $y_{m}$ (black dashed line); the $\pm 3 \sigma_{\varepsilon}$ region .of the measurement (grey area).

By the MCMC and MMSE methods, the posterior modes provide a good one-point estimate of bending stiffness. As expected, when increasing the number of measurements, the mode becomes closer to the "true" value of the parameter. PCE-KF performs satisfactorily when the "true" value is in the high prior probability region, but the results are very poor due to the linearization when $E J_{\text {true }}=m+3 \mathrm{~s}$. However, since the relationship between the uncertain parameter $E J$ and the measurement $w$ is explicit, a linearization of the measurement operator can easily be performed [53] for this specific case by defining a response map $\Phi$ :

$$
\Phi(w(x))=\frac{1}{w(x)} .
$$

When transforming the observations, the measurement error should also be transformed and the gradient of the map should be calculated in order to define the mapped error.

Repeating the updating after the mapping of the response, we notice that the PCE-KF succeeds in identifying the input-as shown in Figure 7 and Tables 6 and 7-where the percentage deviation $\Delta \%$ between $E J_{m}^{\prime}$ and $E J_{\text {true }}$ is reduced with respect to the previous case (Tables 4 and 5). 


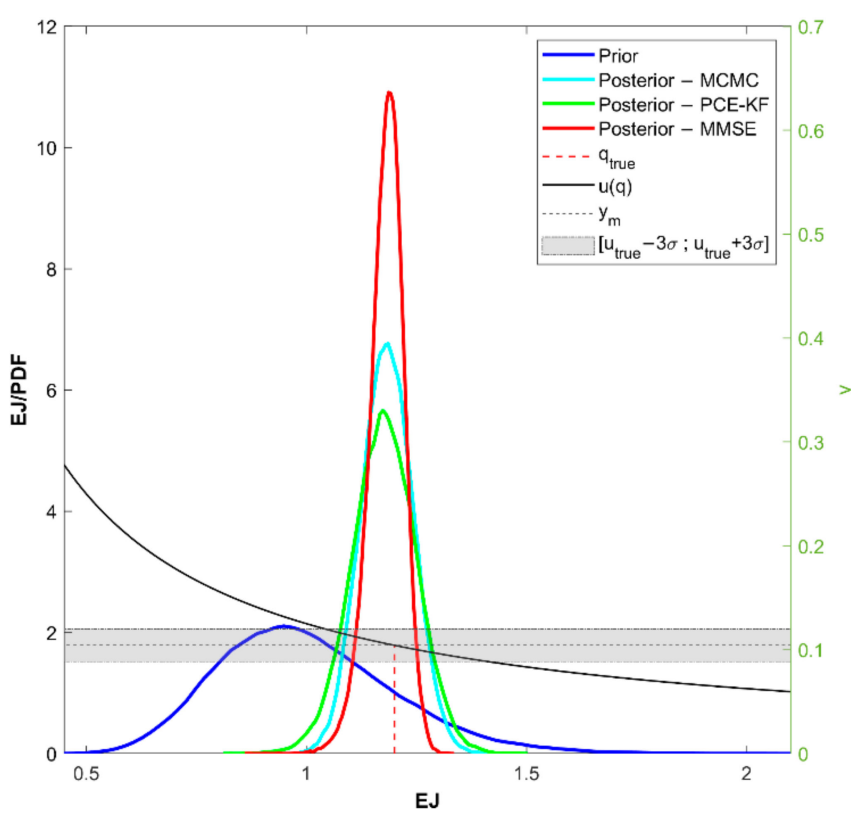

(a)

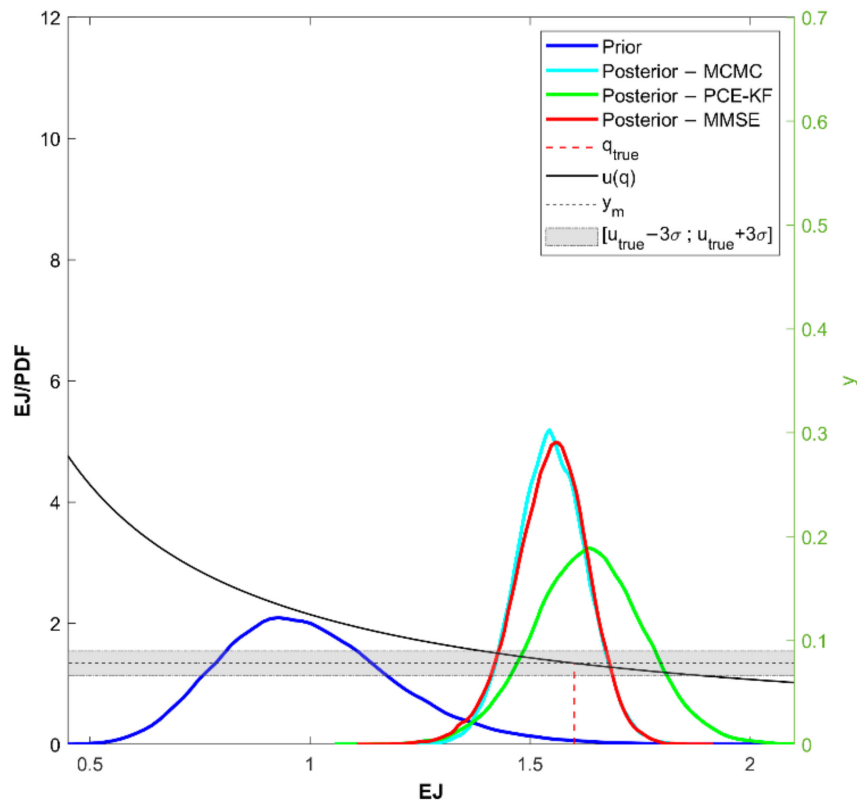

(b)

Figure 7. Comparison of updating procedures after mapping-cantilever beam: (a) $E J_{\text {true }}=m+s=1.2$ and (b) $E J_{\text {true }}=m+3 s=1.6$. In each figure, the following are reported: the prior PDF (in blue); the posterior PDFs obtained via MCMC (in cyan), via PCE-KF (in green) and via NL-MMSE (red solid line); the true value of the parameter $q_{\text {true }}$ (red dashed line); the model response $u(q)$ (black solid line); the measurement $y_{m}$ (black dashed line); the $\pm 3 \sigma_{\varepsilon}$ region of the measurement (grey area).

Finally, we study the impact of the measurement error on the posterior distribution for $E J_{\text {true }}=m+3 \mathrm{~s}$; the variations in percentage error in the different updating procedures, after mapping, are shown in Figure 8. As highlighted in the previous section, since the derivative of the model in $E J_{\text {true }}=m+s$ is greater than the derivative in $E J_{\text {true }}=m+3 \mathrm{~s}$, in the former case, the influence measurement error is smaller than in the latter case. Therefore, the low values of the update errors that we obtain for $q_{\text {true }}: \mu+\sigma$ can be explained not only in terms of better prior knowledge, but also in relation to the minor influence of the measurement error.

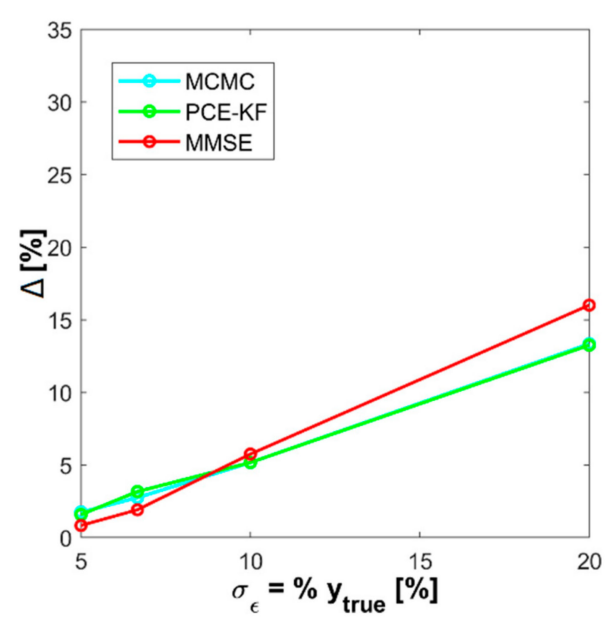

(a)

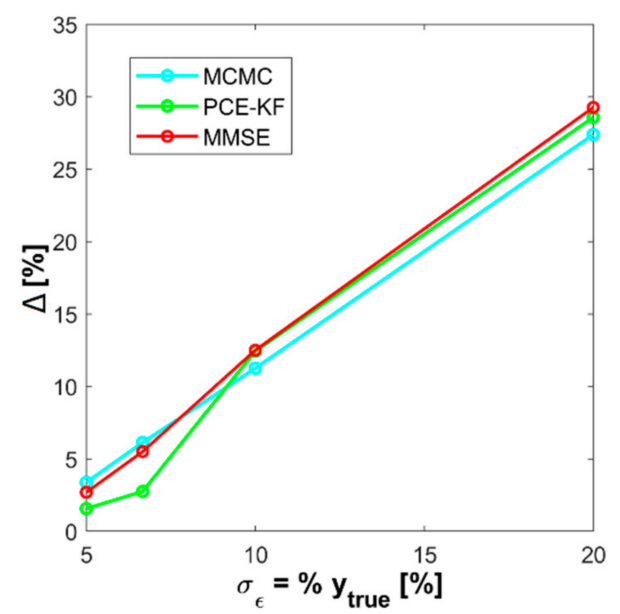

(b)

Figure 8. Percentage error in the updating according to different methods, variations with measurement error: (a) $E J_{\text {true }}=m+s=1.2$ and (b) $E J_{\text {true }}=m+3 s=1.6$. Results are compared for the MCMC method (in cyan), the PCE-KF method (in green) and the NL-MMSE method (in red). 


\subsection{One-Dimensional Dynamic Problem}

We now consider a 1D dynamic problem, which is a single degree of freedom (SDOF) system represented by a cantilever beam with a height $L=1$ and a mass $\boldsymbol{m}=10$ at the free end (Figure 9). Despite being schematic, this simple model of an inverted pendulum is widely used in engineering studies; it can be used, for example, as a simplified reference model to study the structural behavior of a bridge pier.

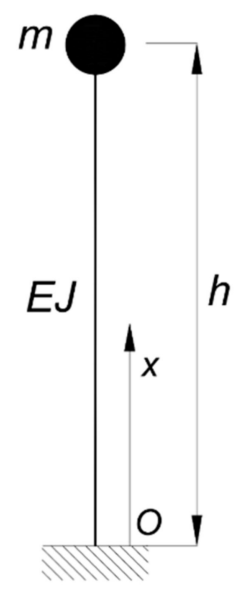

Figure 9. Single degree of freedom system (SDOF).

Let us assume from our engineering expertise that the bending rigidity $E J$ is a random variable described by a lognormal prior distribution with mean $\overline{E J}=1$ and standard deviation $s_{E J}=0.2$. Then, the updating is performed through a measurement of the fundamental natural frequency of the system $f=\frac{\omega}{2 \pi}=\sqrt{\frac{k}{m}} \frac{1}{2 \pi}$ with $k=\frac{3 E J}{h^{3}}$.

As observed in the 1D static problem, MCMC and MMSE can be equivalently used for the parameter identification (see Table 8 and Figure 10). However, in this case, PCE-KF also performs a satisfying update because of the low degree of non-linearity of the response in the measurement region.

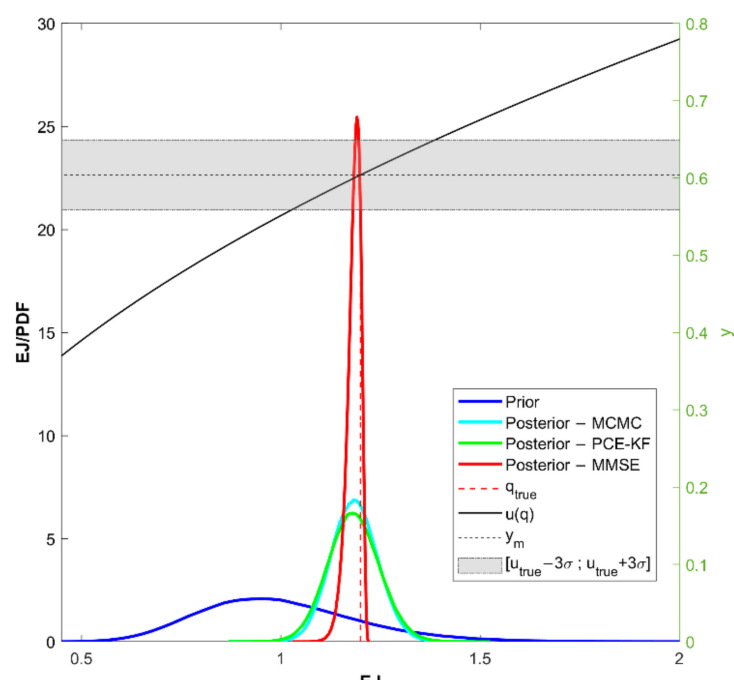

(a)

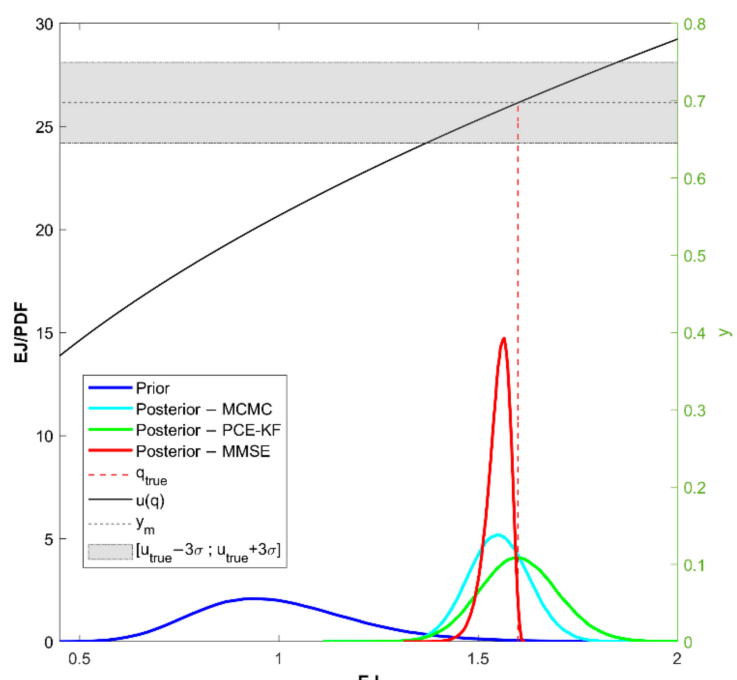

(b)

Figure 10. Comparison of updating procedures-SDOF: (a) $E J_{\text {true }}=\overline{E J}+s_{E J}=1.2$ and (b) $E J_{\text {true }}=\overline{E J}+3 s_{E J}=1.6$. In each figure, the following are reported: the prior PDF (in blue); the posterior PDFs obtained via MCMC (in cyan), via PCE-KF (in green) and via NL-MMSE (red solid line); the true value of the parameter $q_{\text {true }}$ (red dashed line); the model response $u(q)$ (black solid line); the measurement $y_{m}$ (black dashed line); the $\pm 3 \sigma_{\varepsilon}$ region of the measurement (grey area). 
The case in which two parameters, namely $\boldsymbol{E} \boldsymbol{J}$ and $\boldsymbol{m}$, are affected by uncertainty is now considered. When several uncertain parameters are concerned, it is practical to compute the sensitivities of the model, to establish which parameters can be identified. For non-linear models, local sensitivity is evaluated by the gradient, which changes from point to point in the parametric space when a nonlinear model is considered. For such a model, it is beneficial to look at global sensitivities instead. Variance-based local sensitivities, the Sobol indices [62,63], can be computed in a straightforward and computationally efficient way from the PCE surrogate model [64].

Assuming the mass $m$ is a random variable described by a lognormal prior distribution with mean $\bar{m}=10$ and coefficient of variation (COV) equal to $0.2-$ which is the same $\mathrm{COV}$ of that previously considered for the bending rigidity $E J$ - the model shows an equal sensitivity to the two uncertain parameters, making them identifiable. Figure 11 shows the results obtained with different updating procedures.

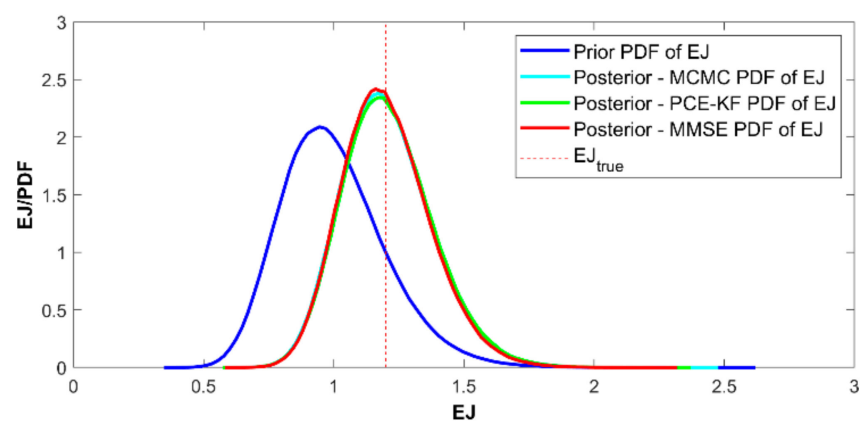

(a)

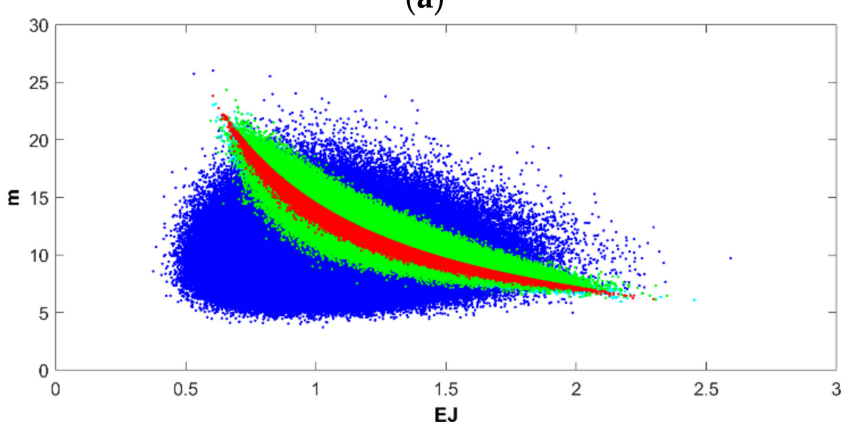

(c)

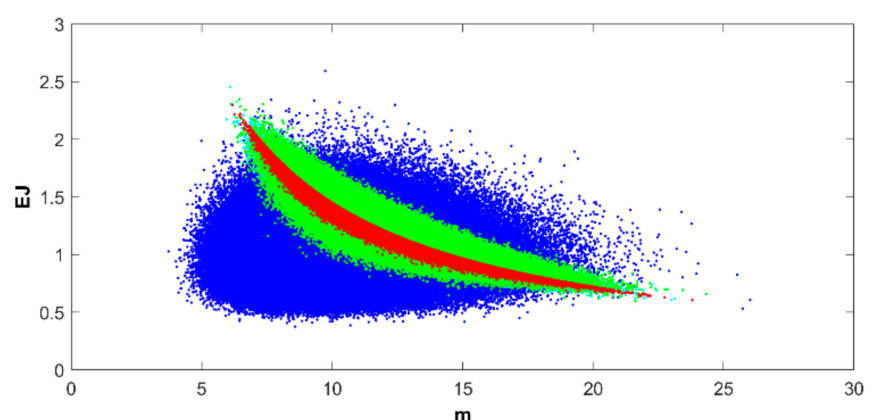

(b)

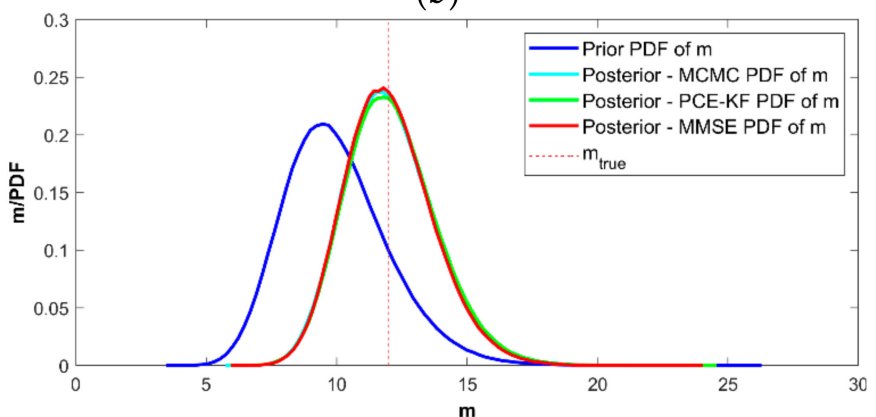

(d)

Figure 11. Comparison of different updating methods with two uncertain parameters-SDOF: (a) prior and marginal posterior $p d f f_{S}$ of $E J,(\mathbf{b})$ scatterplot of the samples of the prior and posterior $p d f s,(\mathbf{c})$ prior and marginal posterior $p d f s$ of $m$ and (d) scatterplot of the samples of the prior and posterior $p d f s$. The prior distributions and the results of the different methods are shown with different colors: prior (in blue), MCMC (in cyan), PCE-KF (in green) and MMSE (in red).

Regarding reducing the uncertainty associated with the mass $m$, i.e., decreasing the coefficient of variation $\left(\mathrm{COV}_{m}\right)$, the sensitivity of the response to the mass decreases and the mass parameter becomes less identifiable compared to stiffness. This fact can be visualized by means of the response surfaces (Figure 12). Sensitivities for each parameter ( $S I_{E J}$ and $S I_{m}$ ) are computed in terms of Sobol indices, which are analytically derived directly from the PCE expansion of the model response [64]. Different values of $\mathrm{COV}_{m}$ are considered $\left(\mathrm{COV}_{m}=0.05,0.1,0.15\right.$ and 0.2$)$; as the sensitivity index for the mass decreases, the dependence of the response surface on the mass parameter weakens. This outcome is graphically shown in Figure 12 by the intersection line of the response surface with the measurement, which becomes almost parallel to the $m$-axis when $S I_{m}$ is reduced, thus making the mass parameter unidentifiable. 


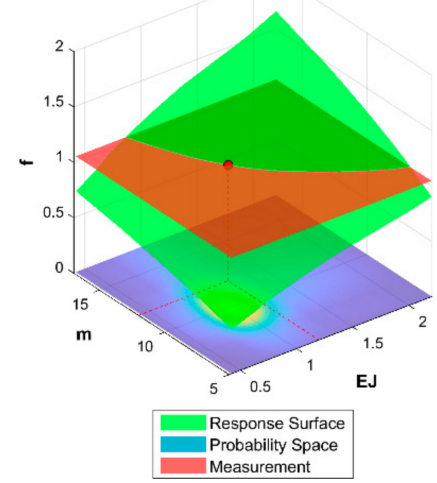

(a)

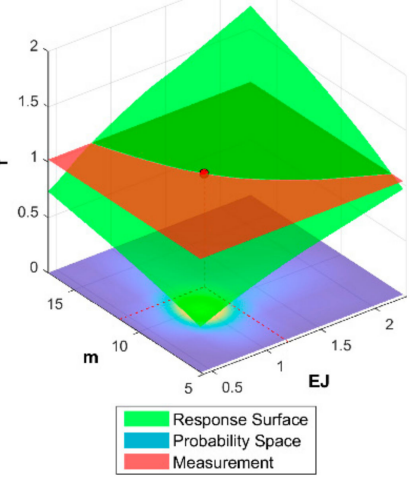

(b)

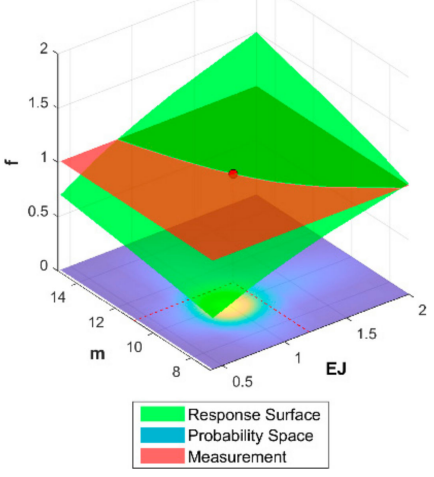

(c)

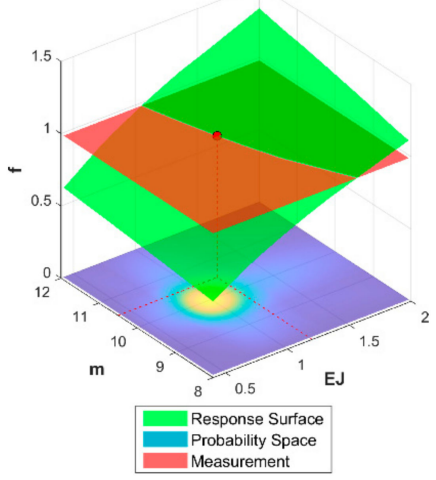

(d)

Figure 12. Comparison of response surfaces with different sensitivities for the parameters-SDOF: (a) COV $m=0.05$, (b) $\mathrm{COV}_{m}=0.1,(\mathbf{c}) \mathrm{COV}_{m}=0.15$ and (d) $\mathrm{COV}_{m}=0.2$.

This evidence is corroborated by the results shown in Figure 13, where the posterior modes and standard deviation of the two parameters are plotted against Sobol indices. Posterior modes for $E J$ and $m$ become closer to the true value (dashed black lines) as the sensitivity of the model, with respect to these parameters, increases; the EJ posterior standard deviation decreases and the $m$ posterior standard deviation increases, as the $m$ prior standard deviation increases due to the increased sensitivity of the response surface to the mass parameter.

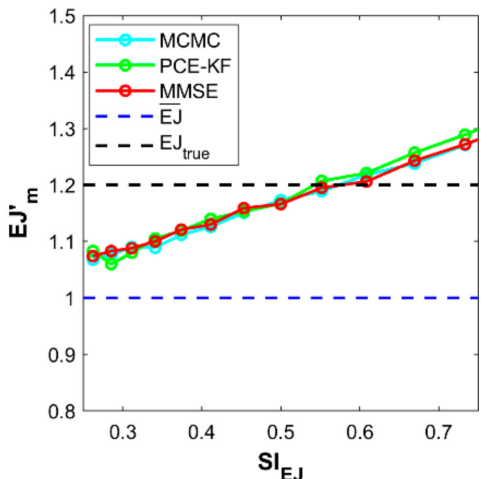

(a)

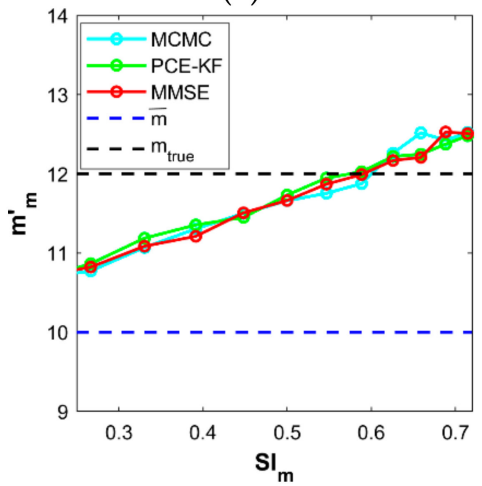

(c)

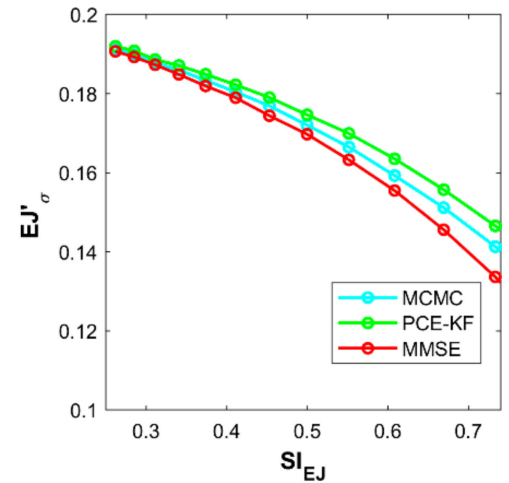

(b)

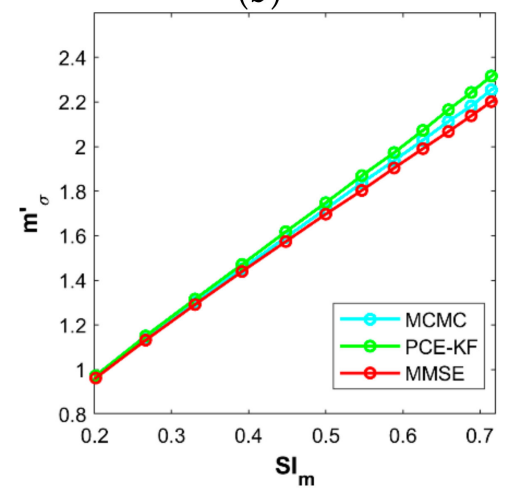

(d)

Figure 13. Posterior modes and standard deviations of $E J$ and $m$ considering the variation in sensitivities $\left(S I_{E J}\right.$ and $S I_{m}$ ): (a) posterior mode $E J_{m}^{\prime}$, (b) posterior standard deviation $E J_{\sigma}^{\prime}$, (c) posterior mode $m_{m}^{\prime}$ and (d) posterior standard deviation $m_{m}^{\prime}$. Results are compared for the MCMC method (in cyan), the PCE-KF method (in green) and the NL-MMSE method (in red). 


\subsection{Real Case Study: Reinforced Concrete Water Tank}

A real case study of a reinforced concrete water tank, already analyzed by the authors in a previous study [20], is now considered in the benchmark study. This water storage facility is a component of a complex infrastructure system and its maintenance requires special attention for fundamental functioning within the network. In order to analyze its structural behavior, the FE model illustrated in Figure 14 is created and, to reduce the uncertainty in the reliability assessment, a calibration of the input parameters is carried out considering different measurements and updating procedures.

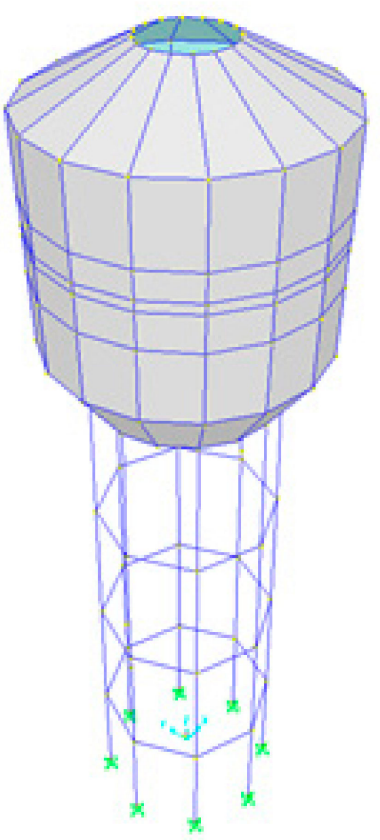

Figure 14. FE model of the concrete water tank.

In a first stage, the modulus of elasticity $E$ is considered as the unique uncertain parameter of the model, which is characterized by a lognormal prior distribution with mean $\bar{E}=42 \mathrm{GPa}$ and standard deviation $s_{E}=10 \mathrm{GPa}$. The updating is carried out by measuring the fundamental natural frequency $f_{1}$, whose measurement error can be described by a normal distribution $N(0,0.02 \mathrm{~Hz})$. The results are shown in Table 9 and Figure 15, confirming what was observed for the SDOF problem. In fact, MCMC and MMSE give good results in terms of posterior modes compared with the true values; PCE$\mathrm{KF}$ is also able to perform a satisfactory update because of the low degree of non-linearity of the response when the measurement is close to the prior mean ( $\bar{E} \pm 2 s_{E}$ region).

The updating of the uncertain parameter is then performed considering the measurement of displacements in two different points (for an extensive description of the experimental campaign, please refer to [20]). The pdf of the measurement error of the displacement is characterized by a standard deviation of $\sigma_{\varepsilon}=0.1 \mathrm{~mm}$. The results are summarized in Table 10. It emerges that larger errors are obtained and the PCE-KF is not able to perform the update due to the increased degree of non-linearity of the response. Linearizing the measurement operator is again a good way to reduce the errors for the PCE-KF, as confirmed by the results in Table 11. 


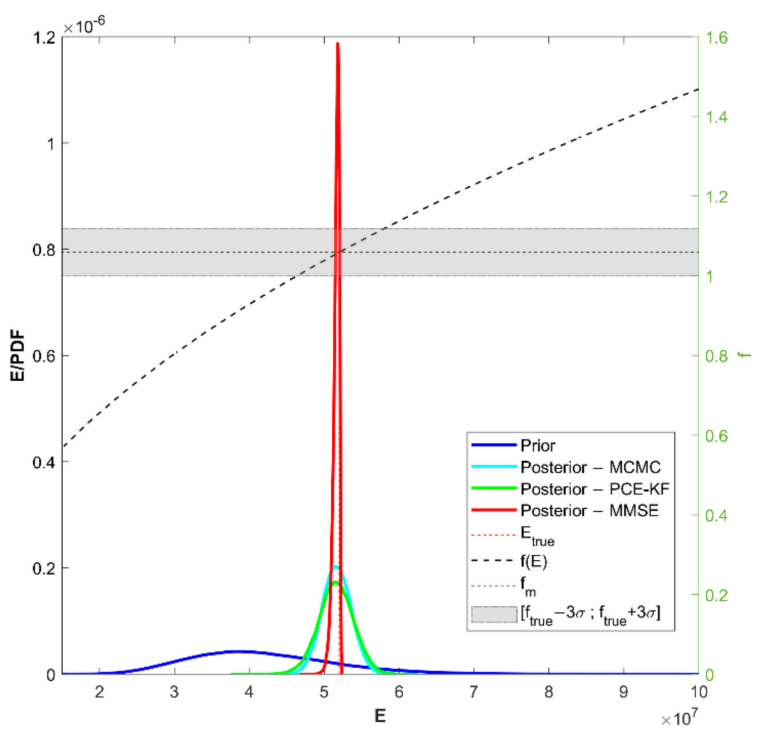

(a)

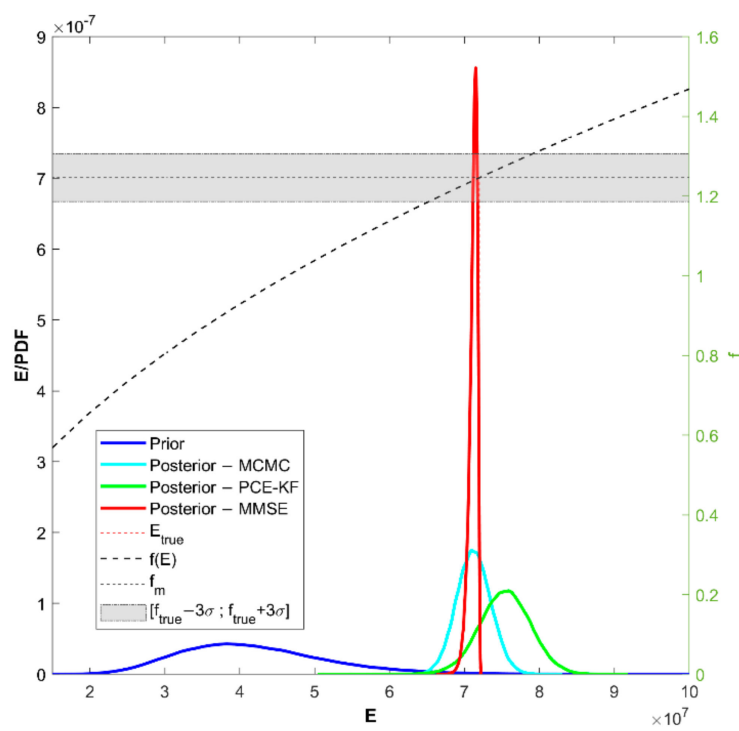

(b)

Figure 15. Comparison of updating procedures-concrete water tank: (a) $E_{\text {true }}=\bar{E}+s_{E}$ and (b) $E_{\text {true }}=\bar{E}+3 s_{E}$. In each figure, the following are reported: the prior PDF (in blue); the posterior PDFs obtained via MCMC (in cyan), via PCE-KF (in green) and via NL-MMSE (red solid line); the true value of the parameter $E_{\text {true }}$ (red dashed line); the model response $f(E)$ (black solid line); the measurement $f_{m}$ (black dashed line); the $\pm 3 \sigma_{\varepsilon}$ region of the measurement (grey area).

The impact of the measurement error on the results of the updating is also investigated by increasing the standard deviation $\sigma_{\varepsilon}$ from 0.1 to $0.5 \mathrm{~mm}$. The results are illustrated in Figure 16 for both cases: $E_{\text {true }}=\bar{E}+s_{E}$ and $E_{\text {true }}=\bar{E}+3 s_{E}$. Similar trends are obtained for the different updating methods with slightly higher values of deviation for the PCE-KF.

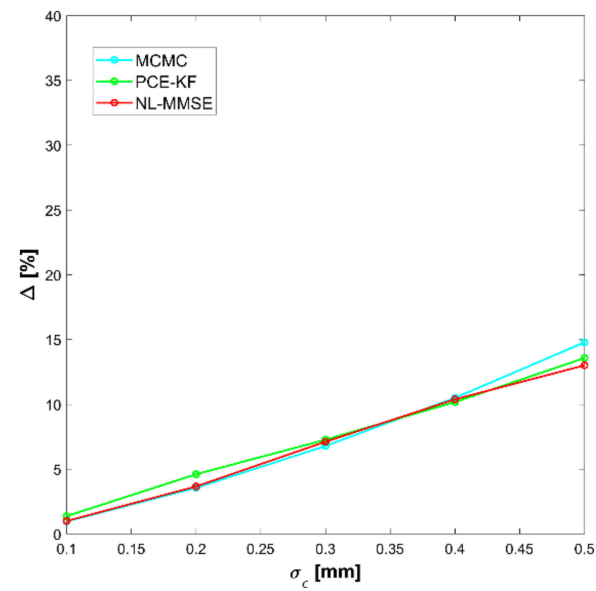

(a)

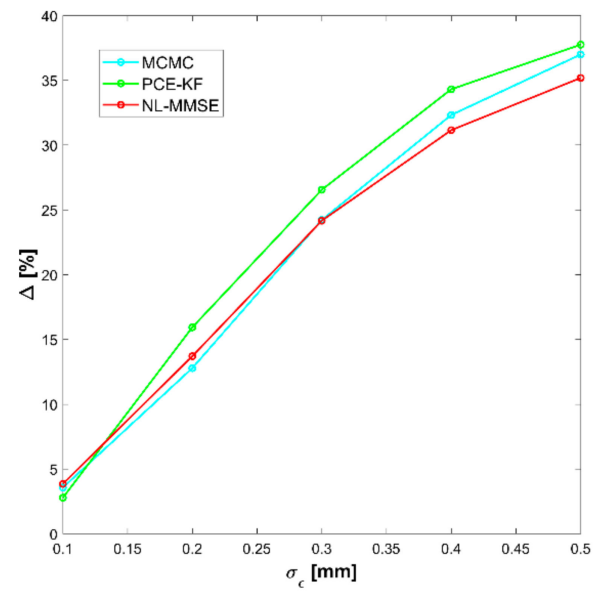

(b)

Figure 16. Percentage deviation in the updating according to different methods, variation with measurement error: (a) $E_{\text {true }}=\bar{E}+s_{E}$ and (b) $E_{\text {true }}=\bar{E}+3 s_{E}$. Results are compared for the MCMC method (in cyan), the PCE-KF method (in green) and the NL-MMSE method (in red).

We assume now that the density $w$ of reinforced concrete is also a random variable, which can be described by a lognormal prior distribution with mean $\bar{w}=24 \mathrm{kN} / \mathrm{m}^{3}$ and standard deviation $s_{w}=1.2 \mathrm{kN} / \mathrm{m}^{3}$. First, the forward problem is solved and the Sobol indices of the random parameters are computed in order to assess their identifiability. Sobol indices are equal to 0.98 for concrete elastic modulus $E$ and to 0.02 for concrete density $w$, denoting the low relevance of concrete density in the computation of the response. This outcome is confirmed by the diagram in Figure 17. 


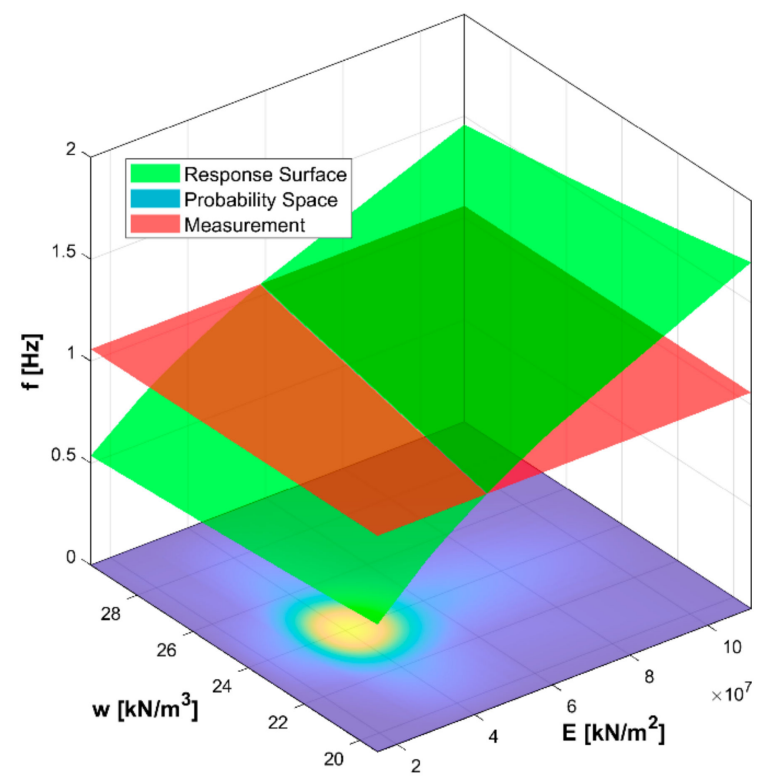

Figure 17. Response surface of the fundamental natural frequency $f$-concrete water tank.

Looking at the figure, it can be observed that the intersection of the response surface (in green) and of the measurement plane is a line (in red) almost parallel to the $w$-axis. Based on these observations, we expect that the identifiable parameter is only the elastic modulus; this conclusion is confirmed by the results illustrated in Figure 18.

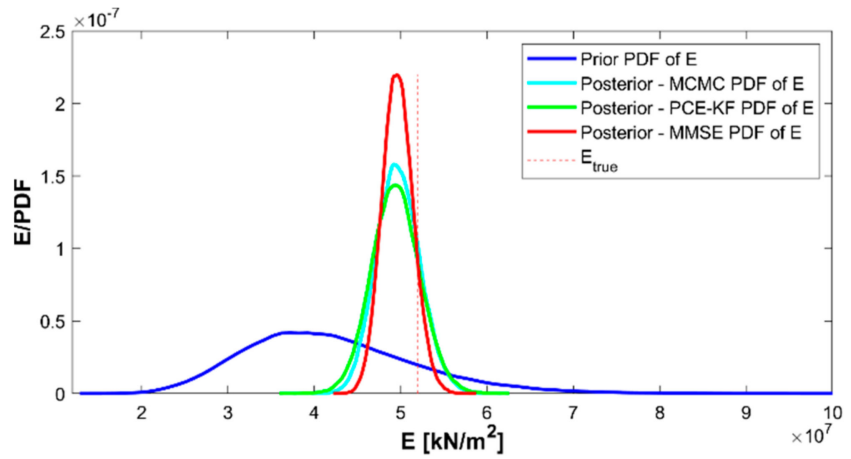

(a)

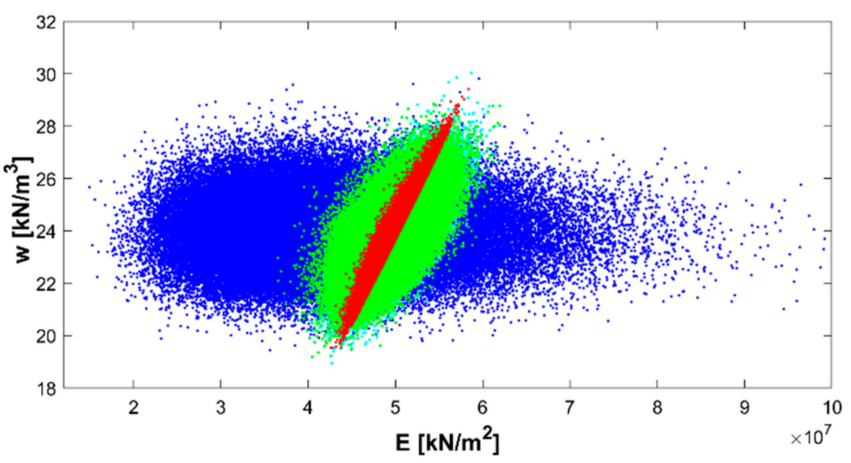

(c)

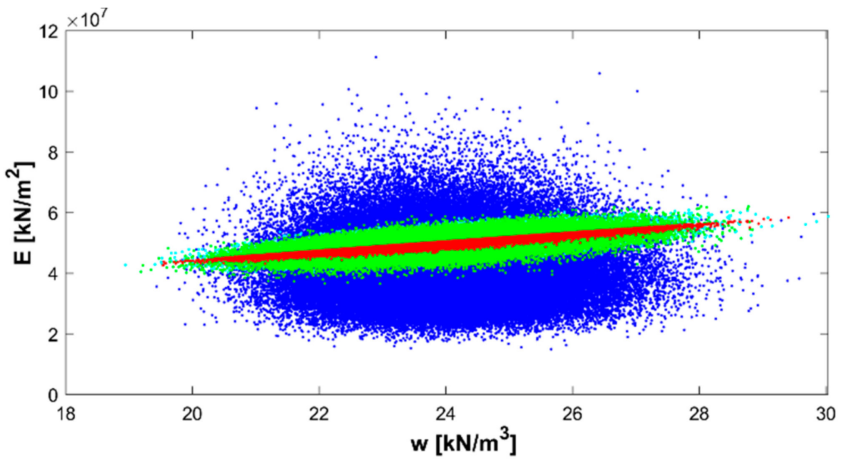

(b)

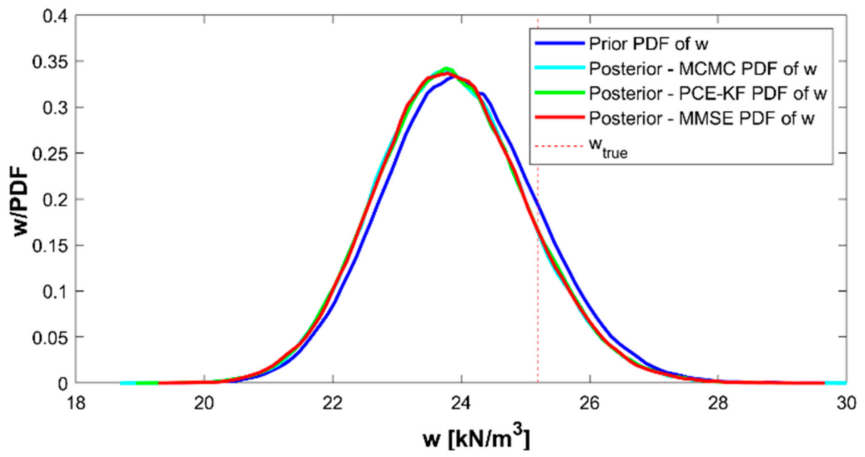

(d)

Figure 18. Comparison of different updating methods with two uncertain parameters-concrete water tank: (a) prior and marginal posterior $p d f f_{S}$ of $E J$, (b) scatterplot of the samples of the prior and posterior $p d f s,(\mathbf{c})$ prior and marginal posterior $p d f s$ of $m$ and (d) scatterplot of the samples of the prior and posterior $p d f s$. The prior distributions and the results of the different methods are shown with different colors: prior (in blue), MCMC (in cyan), PCE-KF (in green) and MMSE (in red). 


\section{Conclusions}

In this paper, three PCE-based stochastic inverse methods-the Markov Chain Monte Carlo (MCMC), the polynomial chaos expansion-based Kalman Filter (PCE-KF) and a method based on the minimum mean square error (MMSE) — are tested on a benchmark of models characterized by an increasing degree of complexity, namely four analytical abstract models, a one-dimensional static model, a one-dimensional dynamic model and an FE model. Meanwhile, important problems commonly encountered in civil, structural and engineering fields are also taken into consideration; most notably, the degree of nonlinearity of a model, the basic engineering knowledge reflected by an adopted prior model, the sensitivity of a model to the uncertain parameters, the information content of the available measurements and the magnitude of the measurement error.

From the presented results, it is possible to draw the following conclusions:

- The PCE-KF struggles to identify the input parameters when the dependence of the measurable quantities is non-linear. This is especially true when the non-linearity is significant and when the "true" value of the parameter lies in a low prior probability region. However, the performance of the method can be improved by defining a mapping function capable of linearizing the model's response, when such a map is available, or when it can be easily envisioned. For example, if the task is to identify the stiffness of a structure by measuring displacements, the flexibility (the inverse of the stiffness) should instead be identified. In more sophisticated highly non-linear FE models, one does not have the knowledge of the magnitude of non-linearity and/or it is difficult to convert the problem to a linear description $[65,66]$. Therefore, after checking nonlinearity by means of uncertainty quantification methods, if the non-linearity is strong, it is recommended to use another method for updating.

- The MCMC is an efficient but brute force method for all investigated models, capturing the bimodal shape of the posterior distribution. However, on the one hand, the method requires a high computational effort; on the other hand, its convergence can be very slow. In addition, the derived posterior is only given in the form of samples. Such a description makes a sequential update difficult when we wish to repeat the update when new measurements become available. Nevertheless, when the goal is to update the structural model only once, in an offline manner, this is a very effective method to tackle the posterior distribution of the uncertain parameters or fields, that can be used in a general way for any civil engineering problems.

- The MMSE represents a good alternative to an MCMC capable of deriving a closed form of the posterior in terms of functional representation. The performance of MMSE was promising for all tested models considered in this paper. Nevertheless, for multimodal posterior distributions, it is often more beneficial to use the MCMC method when the goal is not only to compute the mean of the Bayesian posterior. This method, similar to its linear update version, the Kalman Filter, makes a sequential update of the uncertain parameters or fields possible due to the functional representation of the posterior distribution. A disadvantage of the MMSE method is that it suffers strongly from the curse of dimensionality, which makes it difficult to apply when the dimension of the uncertain parameters is high. Dimension reduction methods may tackle this issue, as described in [24], but could also complicate computations further.

Good knowledge of the engineering problem (i.e., an adequate choice of the prior distribution) is fundamental for the success of the identification process. However, unsatisfactory results due to a poor prior model can be improved by increasing the number of measurements and the information content of the measurement (when possible). The evaluation of the Sobol indices is fundamental for assessing the identifiability of the parameters, while also offering significant information about the experiment that should be carried out. Based on the sensitivities, the most suitable output to be measured can be selected and the location of the sensors is optimized [67]. It has been shown how higher derivatives in the measurement region imply a higher sensitivity of the response to the 
parameter variations, thus facilitating parameter identification and reducing the effect of measurement errors on updating.

The ultimate scope of this paper is to promote a critical approach for the use of structural identification procedures, as well as to shed light on the influence of the most important aspects of inverse problems usually encountered in civil engineering in terms of updating results.

Author Contributions: Conceptualization, F.L., F.M., N.F. and P.C.; methodology, F.L., F.M., N.F. and P.C.; software, F.L., F.M., N.F. and P.C.; validation, F.L., F.M., N.F. and P.C.; investigation, F.L., F.M., N.F. and P.C.; writing—original draft preparation, F.L., F.M., N.F. and P.C.; writing-review and editing, F.L., F.M., N.F. and P.C.; visualization, F.L., F.M., N.F. and P.C. All authors have read and agreed to the published version of the manuscript.

Funding: This research received no external funding.

Institutional Review Board Statement: Not applicable.

Informed Consent Statement: Not applicable.

Data Availability Statement: The data presented in this study are available on request from the corresponding author.

Conflicts of Interest: The authors declare no conflict of interest.

\section{Appendix A}

As mentioned in the paper, a parallelized version of the MCMC algorithm was used for the calculations. The key idea of the algorithm is to run $T$ multiple chains of $N$ samples in parallel; thus, the $z_{\mathrm{i}}$ samples of the posterior distribution are obtained according to the following steps:

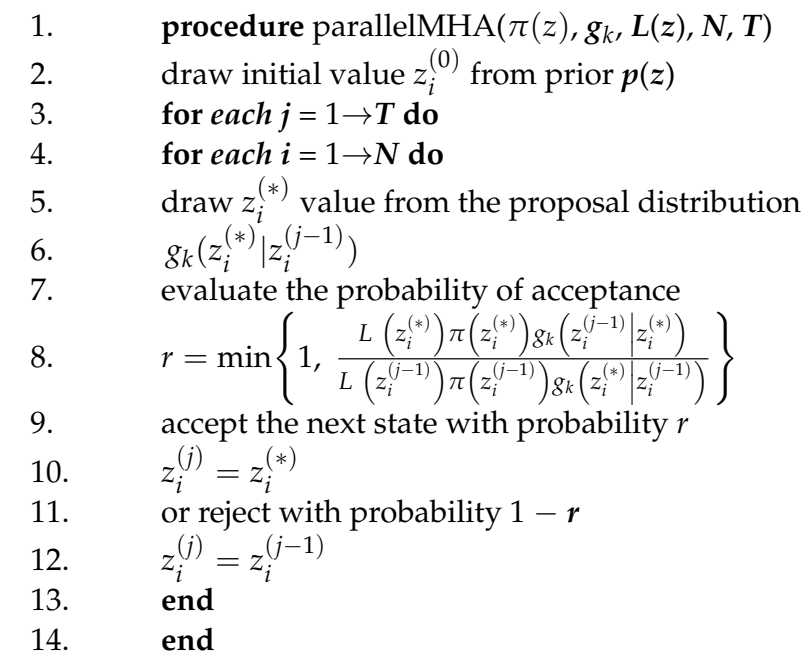

\section{References}

1. Çatbaş, N.F.; Kijewski-Correa, T.; Aktan, A.E. Structural Identification of Constructed Systems; American Society of Civil Engineers (ASCE) Book Series; ASCE: Preston, VA, USA, 2013.

2. Croce, P.; Formichi, P.; Landi, F. Influence of Reinforcing Steel Corrosion on Life Cycle Reliability Assessment of Existing R.C. Buildings. Buildings 2020, 10, 99. [CrossRef]

3. Beconcini, M.L.; Croce, P.; Formichi, P.; Landi, F.; Puccini, B. Experimental Evaluation of Shear Behavior of Stone Masonry Wall. Materials 2021, 14, 2313. [CrossRef] [PubMed]

4. Croce, P.; Beconcini, M.L.; Formichi, P.; Landi, F.; Puccini, B.; Zotti, V. Bayesian Methodology for Probabilistic Description of Mechanical Parameters of Masonry Walls. ASCE ASME J. Risk Uncertain. Eng. Syst. Part A Civ. Eng. 2021, 7, 04021008. [CrossRef]

5. Marwala, T. Finite-Element-Model Updating Using Computational Intelligence Techniques; Springer, London Limited: London, UK, 2010.

6. Schlune, H.; Plos, M.; Gylltoft, K. Improved bridge evaluation through finite element model updating using static and dynamic measurements. Eng. Struct. 2009, 31, 1477-1485. [CrossRef] 
7. Matthies, H.G.; Zander, E.K.; Rosic, B.V.; Litvinenko, A.; Pajonk, O. Inverse Problems in a Bayesian Setting. In Computational Methods for Solids and Fluids; Ibrahimbegovic, A., Ed.; Springer International Publishing: Cham, Switzerland, 2016 ; pp. $245-286$. [CrossRef]

8. Ang, H.S.; Tang, W.H. Probability Concepts in Engineering: Emphasis on Applications to Civil and Environmental Engineering, 2nd ed.; John Wiley and Sons: Hoboken, NJ, USA, 2002; Volume 1.

9. Bayes, T.; Price, R. (An essay towards solving a problem in the doctrine of chances. Phil. Trans. 1753, 53, 370-418. [CrossRef]

10. Beck, J.L. Statistical system identification of structures. In Proceedings of the 5th International Conference on Structural Safety and Reliability, San Francisco, CA, USA, 7-11 August 1989.

11. Beck, J.L.; Katafygiotis, L.S. Updating of a model and its uncertainties utilizing dynamic test data. In Computational Stochastic Mechanics; Spanos, P.D., Brebbia, C.A., Eds.; Springer: Dordrecht, The Netherlands, 1991; pp. 125-136. [CrossRef]

12. Beck, J.L.; Katafygiotis, L.S. Updating models and their uncertainties. I: Bayesian statistical framework. J. Eng. Mech. 1998, 124, 455-461. [CrossRef]

13. Katafygiotis, L.S.; Beck, J.L. Updating models and their uncertainties. II: Model identifiability. J. Eng. Mech. 1998, $124,463-467$. [CrossRef]

14. Simoen, E.; Moaveni, B.; Conte, J.P.; Lombaert, G. Uncertainty Quantification in the Assessment of Progressive Damage in a 7-Story Full-Scale Building Slice. J. Eng. Mech. 2013, 139, 1818-1830. [CrossRef]

15. Nagel, J.B.; Mojsilovic, N.; Sudret, B. Bayesian Assessment of the Compressive Strength of Structural Masonry. In Proceedings of the 12th International Conference on Applications of Statistics and Probability in Civil Engineering (ICASP12), Vancouver, BC, Canada, 12-15 July 2015; University of British Columbia: Vancouver, BC, Canada, 2015. [CrossRef]

16. Conde, B.; Eguía, P.; Stavroulakis, G.E.; Granada, E. Parameter identification for damaged condition investigation on masonry arch bridges using a Bayesian approach. Eng. Struct. 2018, 172, 275-284. [CrossRef]

17. Yuen, K. Bayesian Methods for Structural Dynamics and Civil Engineering; John Wiley \& Sons (Asia): Singapore, 2010.

18. Simoen, E.; Lombaert, G. Bayesian Parameter Estimation. In Identification Methods for Structural Health Monitoring; Chatzi, E., Papadimitriou, C., Eds.; Springer: Cham, Switzerland, 2016; pp. 89-115. [CrossRef]

19. Huang, Y.; Shao, C.; Wu, B.; Beck, J.L.; Li, H. State-of-the-art review on Bayesian inference in structural system identification and damage assessment. Adv. Struct. Eng. 2018, 22, 1329-1351. [CrossRef]

20. Marsili, F.; Croce, P.; Friedman, N.; Formichi, P.; Landi, F. Seismic Reliability Assessment of a Concrete Water Tank Based on the Bayesian Updating of the Finite Element Model. ASCE ASME J. Risk Uncertain. Eng. Syst. Part B Mech. Eng. 2017, 3, 1-14. [CrossRef]

21. Sevieri, G.; Andreini, M.; De Falco, A.; Matthies, H.G. Concrete gravity dams model parameters updating using static measurements. Eng. Struct. 2019, 196, 109231. [CrossRef]

22. Pepi, C.; Gioffré, M.; Grigoriou, M.D.; Matthies, H.G. Bayesian updating of cable stayed footbridge model parameters using dynamic measurements. In Proceedings of the 3rd ECCOMAS Thematic Conference on Uncertainty Quantification in Computational Sciences and Engineering, Crete, Greece, 24-26 June 2019; Papadrakakis, M., Papadopoulos, V., Stefanou, G., Eds.; Ecommas Proceedia: Crete, Greece, 2019; pp. 330-342. [CrossRef]

23. Kurent, B.; Friedman, N.; Brank, B. On (Bayesian) Finite Element Model Updating of Civil Engineering Structures by Using Modal Features. In Proceedings of the ECCOMAS MSF 2021 Thematic 5th Conference 'Multiscale Computational Methods in Solids and Fluids', Split, Croatia, 30 June-2 July 2021.

24. Friedman, N.; Zoccarato, E.; Zander, E.; Matthies, H.G. A Worked-out Example of Surrogate-based Bayesian Parameter and Field Identification Methods. In Bayesian Methods for the Analysis of Engineering Systems; Chiachio Ruano, J., Chiachio Ruano, M., Sankararaman, S., Eds.; CRC Press: Boca Raton, FL, USA, 2021.

25. Croce, P.; Formichi, P.; Landi, F. A Bayesian hierarchical model for climatic loads under climate change. In Proceedings of the 3rd ECCOMAS Thematic Conference on Uncertainty Quantification in Computational Sciences and Engineering, Crete, Greece, 24-26 June 2019; pp. 298-308. [CrossRef]

26. Tarantola, A. Inverse Problem Theory and Methods for Model Parameter Estimation; Society for Industrial and Applied Mathematics (SIAM): Philadelphia, PA, USA, 2005. [CrossRef]

27. Hastings, W.K. Monte Carlo sampling methods using Markov chains and their applications. Biometrika 1970, 57, 97-109. [CrossRef]

28. Neal, R.N. Probabilistic Inference Using Markov Chain Monte Carlo Methods; Technical Report CRG-TR-93-1; Department of Computer Science, University of Toronto: Toronto, ON, Canada, 1993.

29. Bolstad, W.M. Understanding Computational Bayesian Statistics; John Wiley \& Sons: Hoboken, NJ, USA, 2010.

30. Berg, B.A. Introduction to Markov Chain Monte Carlo Simulations and their Statistical Analysis. In Markov Chain Monte Carlo; Kendall, W.S., Liang, F., Wang, J.S., Eds.; Lecture Notes Series; Institute for Mathematical Sciences; National University of Singapore: Singapore, 2005; Volume 7.

31. Wiener, N. The homogeneous chaos. Am. J. Math. 1938, 60, 897-936. [CrossRef]

32. Marzouk, Y.M.; Najm, H.N.; Rahn, L.A. Stochastic spectral methods for efficient Bayesian solution of inverse problems. J. Comput. Phys. 2007, 224, 560-586. [CrossRef]

33. Sudret, B. Polynomial chaos expansions and stochastic finite element methods. In Risk and Reliability in Geotechnical Engineering; Phoon, K., Ching, J., Eds.; CRC Press: Boca Raton, FL, USA, 2015; pp. 265-300. 
34. Ghanem, R.; Red-Horse, J. Polynomial Chaos: Modelling, Estimation, and Approximation. In Handbook of Uncertainty Quantification; Ghanem, R., Higdon, D., Owhadi, H., Eds.; Springer: Cham, Switzerland, 2017; pp. 521-551. [CrossRef]

35. Marsili, F.; Friedman, N.; Croce, P. Parameter Identification via gPCE-based Stochastic Inverse Methods for Reliability Assessment of Existing Structures. In Proceedings of the International Probabilistic Workshop 2015, Liverpool, UK, 4-6 November 2015.

36. Pandurangan, R.; Chaudhuri, A.; Gupta, S. The use of polynomial chaos for parameter identification from measurements in nonlinear dynamical systems. ZAMM J. Appl. Math. Mech./Zeitschrift Für Angewandte Mathematik und Mechanik 2015, 95, 1372-1392. [CrossRef]

37. Spiridonakos, M.D.; Chatzi, E.N.; Sudret, B. Polynomial Chaos Expansion Models for the Monitoring of Structures under Operational Variability. ASCE ASME J. Risk Uncertain. Eng. Syst. Part A Civ. Eng. 2016, 2, B4016003. [CrossRef]

38. Marsili, F.; Friedman, N.; Croce, P.; Formichi, P.; Landi, F. On Bayesian identification methods for the analysis of existing structures. In IABSE Congress Stockholm, 2016: Challenges in Design and Construction of an Innovative and Sustainable Built Environment; IABSE: Zurich, Switzerland, 2016; ISBN 978-385748144-4.

39. Sevieri, G.; De Falco, A. Dynamic structural health monitoring for concrete gravity dams based on the Bayesian inference. J. Civ. Struct. Health Monit. 2020, 10, 235-250. [CrossRef]

40. Adeli, E.; Rosic, B.; Matthies, H.G.; Reinstadler, S. Effect of Load Path on Parameter Identification for Plasticity Models using Bayesian Methods. In Quantification of Uncertainty: Improving Efficiency and Technology; Lecture Notes in Computational Science and Engineering; D’Elia, M., Gunzburger, M., Rozza, G., Eds.; Springer: Cham, Switzerland, 2017; Volume 137, pp. 1-13. [CrossRef]

41. Adeli, E.; Rosic, B.; Matthies, H.G.; Reinstädler, S.; Dinkler, D. Comparison of Bayesian Methods on Parameter Identification for a Viscoplastic Model with Damage. Metals 2020, 10, 876. [CrossRef]

42. Croce, P.; Marsili, F.; Beconcini, M.L.; Muzzi, M.; Rosso, E. Increasing the Robustness of the Bayesian Analysis. In Safety, Robustness and Condition Assessment of Structures-IABSE Workshop on Safety Robustness and Condition Assessment of Structures; IABSE: Zurich, Switzerland, 2015; pp. 194-201. [CrossRef]

43. Beconcini, M.L.; Croce, P.; Marsili, F.; Muzzi, M.; Rosso, E. Probabilistic reliability assessment of a heritage structure under horizontal loads. Probabilistic Eng. Mech. 2016, 45, 198-211. [CrossRef]

44. Croce, P.; Landi, F.; Formichi, P. Probabilistic seismic assessment of masonry buildings. Buildings 2019, 9, 237. [CrossRef]

45. Kalman, R.; Bucy, R. New results in the linear prediction and filter theory. Trans. ASME J. Basic Eng. 1961, 83, 95-108. [CrossRef]

46. Blanchard, E.D.; Sandu, A.; Sandu, C. A polynomial chaos-based Kalman filter approach for parameter estimation of mechanical systems. J. Dyn. Syst. Meas. Control 2010, 132, 061404. [CrossRef]

47. Matthies, H.G.; Zander, E.; Rosic, B.V.; Litvinenko, A. Parameter estimation via conditional expectation: A Bayesian inversion. Adv. Model. Simul. Eng. Sci. 2016, 3, 24. [CrossRef]

48. Zander, E.K. Nonlinear Minimum Mean Square Error Estimation; Internal Report; Institute of Scientific Computing, Technische Universität Braunschweig: Braunschweig, Germany, 2013.

49. Zander, E.; Friedman, N.; Matthies, H.G. Parameter Identification Based on Conditional Expectation. In Bayesian Methods for the Analysis of Engineering Systems; Chiachio Ruano, J., Chiachio Ruano, M., Sankararaman, S., Eds.; CRC Press: Boca Raton, FL, USA, 2021.

50. Landi, F.; Marsili, F.; Friedman, N.; Croce, P. A comparison of stochastic inverse methods with sampling and functional-based linear and non-linear update procedures. Beton und Stahlbetonbau 2018. [CrossRef]

51. Xiu, D. Numerical Methods for Stochastic Computations; Princeton University Press: Princeton, NJ, USA, 2010.

52. Tiernay, L. Markov chains for exploring posterior distribution. Ann. Stat. 1994, 22, 1701-1728. [CrossRef]

53. Rosic, B.; Sykora, J.; Pajonk, O.; Kucerova, A.; Matthies, H.G. Comparison of Numerical Approaches to Bayesian Updating; Informatikbericht 2014; Technische Universität Braunschweig: Braunschweig, Germany, 2014.

54. Evensen, G. Data Assimilation. The Ensemble Kalman Filter; Springer: Berlin/Heidelberg, Germany, 2009.

55. Bertsekas, D.P.; Tsitsiklis, J.N. Introduction to Probability; Lecture Notes, Course 6.041-6.431; Massachusetts Institute of Technology: Cambridge, MA, USA, 2010.

56. Rosić, B.; Kucerova, A.; Syjora, J.; Pajonk, O.; Litvinenko, A.; Matthies, H.G. Parameter identification in a probabilistic setting. Eng. Struct. 2013, 50, 179-196. [CrossRef]

57. Ernst, O.G.; Sprungk, B.; Starkloff, H.J. Bayesian Inverse Problems and Kalman Filters. In Extraction of Quantifiable Information from Complex Systems; Lecture Notes in Computational Science and Engineering; Dahlke, S., Dahmen, W., Griebel, M., Hackbusch, W., Ritter, K., Schneider, R., Schwab, C., Yserentant, H., Eds.; Springer: Cham, Switzerland, 2014; Volume 102, pp. 133-159. [CrossRef]

58. Zander, E. A Matlab/Octave Toolbox for Stochastic Galerkin Methods. 2020. Available online: https://github.com/ezander/ sglib-testing (accessed on 16 September 2021).

59. Vondrejc, J.; Matthies, H.G. Accurate Computation of Conditional Expectation for Highly Non-Linear Problems. SIAM/ASA J. Uncertain. Quantif. 2019, 7, 1349-1368. [CrossRef]

60. Tomovic, R.; Vukobratovic, M. General Sensitivity Theory; Elsevier: New York, NY, USA, 1972.

61. Ghanem, R.G.; Spanos, P.D. Stochastic Finite Elements: A Spectral Approach; Springer: New York, NY, USA, 2001.

62. Saltelli, A.; Sobol, I. About the use of rank transformation in sensitivity of model output. Reliab. Eng. Syst. Saf. 1995, 50, 225-239. [CrossRef] 
63. Sobol, I. Global sensitivity indices for nonlinear mathematical models and their Monte Carlo estimates. Math. Comput. Simul. 2001, 55, 271-280. [CrossRef]

64. Sudret, B. Global sensitivity analysis using polynomial chaos expansions. Reliab. Eng. Syst. Saf. 2008, 93, 964-979. [CrossRef]

65. Croce, P.; Beconcini, M.L.; Formichi, P.; Cioni, P.; Landi, B.; Mochi, C.; Giuri, R. Influence of mechanical parameters on nonlinear static analysis of masonry walls: A relevant case study. Procedia Struct. Integr. 2018, 11, 331-338. [CrossRef]

66. Beconcini, M.L.; Cioni, P.; Croce, P.; Formichi, P.; Landi, F.; Mochi, C. Non-linear static analysis of masonry buildings under seismic actions. International Institute of Informatics and Systemics. In Proceedings of the 22th World Multi-Conference on Systemics, Cybernetics and Informatics, Orlando, FL, USA, 8-11 July 2018; pp. 126-131, IMSCI 2018. Available online: http:/ / www.iiisci.org/Journal/CV\$/sci/pdfs/EA239AY18.pdf (accessed on 15 October 2021).

67. Janouchová, E.; Kučerová, A. Competitive comparison of optimal designs of experiments for sampling-based sensitivity analysis. Comput. Struct. 2013, 124, 47-60. [CrossRef] 